SLAC-PUB-15591

\title{
Induced Electroweak Symmetry Breaking and Supersymmetric Naturalness
}

\author{
Jamison Galloway ${ }^{a}$, Markus Luty ${ }^{b},{\text { Yuhsin } \text { Tsai }^{b} \text {, Yue Zhao }}^{c}$ \\ ${ }^{a}$ Dipartimento di Fisica, Università di Roma "La Sapienza" \\ and INFN Sezione di Roma, I-00185 Roma \\ ${ }^{b}$ Physics Department, University of California Davis \\ Davis, California 95616 \\ ${ }^{c} S L A C$, Stanford University \\ Menlo Park, California 94025
}

\begin{abstract}
In this paper we study a new class of supersymmetric models that can explain a $125 \mathrm{GeV}$ Higgs without fine-tuning. These models contain additional 'auxiliary Higgs' fields with large tree-level quartic interaction terms but no Yukawa couplings. These have electroweak-breaking vacuum expectation values, and contribute to the VEVs of the MSSM Higgs fields either through an induced quartic or through an induced tadpole. The quartic interactions for the auxiliary Higgs fields can arise from either $D$ terms or $F$-terms. The tadpole mechanism has been previously studied in strongly-coupled models with large $D$-terms, referred to as 'superconformal technicolor.' The perturbative models studied here preserve gauge coupling unification in the simplest possible way, namely that all new fields are in complete $S U(5)$ multiplets. The models are consistent with the observed properties of the $125 \mathrm{GeV}$ Higgs-like boson as well as precision electroweak constraints, and predict a rich phenomenology of new Higgs states at the weak scale. The tuning is less than $10 \%$ in almost all of the phenomenologically allowed parameter space. If electroweak symmetry is broken by an induced tadpole, the cubic and quartic Higgs self-couplings are significantly smaller than in the standard model.
\end{abstract}




\section{Introduction}

The discovery of a Higgs-like particle with mass near $125 \mathrm{GeV}$ at the LHC represents a major advance in our understanding of electroweak symmetry breaking [1,2]. The couplings of this state to the $W$ and $Z$ are close to that of a standard model (SM) Higgs, providing direct evidence that this state is the dominant excitation of the condensate that breaks electroweak symmetry. Even though the couplings of this state are compatible with those of a SM Higgs, there is still room for significant mixing with other Higgs states and/or compositeness of the Higgs at higher scales [3, 4].

The $125 \mathrm{GeV}$ Higgs-like particle is a mixed blessing for supersymmetry. A light Higgs is a hallmark of supersymmetry, but supersymmetric models generally predict a Higgs lighter than $125 \mathrm{GeV}$. In the MSSM, SUSY relates the maximal tree-level Higgs quartic coupling to the electroweak gauge couplings via $\lambda=\frac{1}{8}\left(g^{2}+g^{\prime 2}\right)$. This leads to the tree-level Higgs mass bound $m_{h}<m_{Z}$, which was already ruled out by LEP. Explanations for the observed mass of the Higgs in SUSY have focussed on additional contributions to the Higgs quartic:

- MSSM: Top/stop loops can generate a large quartic [5 10]. However, the same loops also generate a large Higgs quadratic term, resulting in tuning at the level of at least $1 \%$.

- NMSSM: The superpotential coupling $\lambda S H_{u} H_{d}$ gives an additional contribution to the Higgs quartic that can alleviate the naturalness problem 11 14. Taking $\lambda$ as large as possible consistent with perturbativity below the GUT scale improves naturalness relative to the MSSM, but improved naturalness is obtained for larger $\lambda$ [15 19]. See 20, 21] for discussions in light of the Higgs discovery.

- Non-decoupling D-terms: New gauge interactions broken at the weak scale can give additional contributions to the Higgs quartic without tuning [22,23]. These require significant additional matter content to maintain gauge coupling unification.

- Fat Higgs: Compositeness of the NMSSM Higgs fields above the weak scale can explain why couplings like the $\lambda$ coupling of the NMSSM are large at the weak scale without Landau poles below the GUT scale [24,25]. These models also require significant additional matter content to maintain gauge coupling unification.

Overall, there seems to be a trade-off between naturalness and simplicity, leading a number of authors to investigate the possibility that SUSY is not natural [26 31]. 


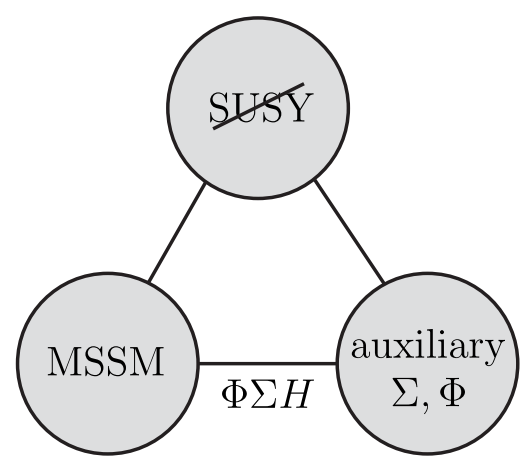

Fig. 1. Schematic structure of the models. The auxiliary Higgs sector contains electroweak doublets $\Sigma$ and electroweak singlets $\Phi$. These interact with the Higgs fields $H$ of the MSSM via superpotential couplings and $A$ terms of the form $\Phi \Sigma H$.

In this paper, we consider a different approach to Higgs naturalness, illustrated schematically in Fig. 1. The idea is that there is an additional sector containing 'auxiliary Higgs' fields with large quartic self-interactions, but no Yukawa couplings. These couple to the MSSM Higgs fields via superpotential couplings and soft SUSY breaking terms, and therefore contribute to the Higgs potential. The auxiliary Higgs fields have VEVs that break electroweak symmetry, so the observed light Higgs is a mixture of the MSSM Higgs and auxiliary Higgs fields. In different limits, the dominant effect on the light Higgs can be viewed as an induced quartic interaction or an induced tadpole. The latter mechanism was proposed in Ref. [32, 33], which considered models where the auxiliary Higgs fields were composites arising from a strong superconformal sector. The mechanism was therefore called 'superconformal technicolor' (see also related work in $34-36]$ ). In this paper we construct perturbative models of this mechanism, which we call 'induced EWSB'.

The large quartic interactions for the auxiliary Higgs fields can arise from new gauge interactions $(D$-terms) and/or new superpotential interactions $(F$-terms). In the case of $D$-terms, this requires that the auxiliary Higgs fields be charged under a new gauge group that is broken at the $\mathrm{TeV}$ scale. The new gauge couplings can easily be stronger than the electroweak gauge couplings at the $\mathrm{TeV}$ scale, so the tree-level auxiliary Higgs quartic can be significantly larger than the tree-level Higgs quartic in the MSSM. In the $F$-term models, the auxiliary Higgs quartic arises from a superpotential coupling $\lambda S \Sigma_{u} \Sigma_{d}$ between a singlet $S$ and auxiliary Higgs doublets $\Sigma_{u, d}$. This can be somewhat larger than the analogous coupling in the NMSSM because $\Sigma_{u, d}$ do not have Yukawa couplings. 
Precision gauge coupling unification can be incorporated in these models in a very simple way: all fields beyond the MSSM can come in complete $S U(5)$ multiplets. For the $D$-term models, VEVs of the auxiliary Higgs fields give rise to mixing between the gauge bosons of the new gauge interactions and those of the electroweak group. This is naturally small if the breaking scale of the new gauge group is sufficiently large, which is in any case required by precision electroweak constraints.

We now describe how the auxiliary Higgs fields improve naturalness of electroweak symmetry breaking. There are two different limits that can be simply understood by integrating out a heavy Higgs multiplet. In the 'induced quartic' limit, the dominant effect is a contribution to the quartic of the light Higgs. In the 'induced tadpole' limit, it is a tadpole for the light Higgs.

We can exhibit these limits in a simplified model with two Higgs doublets $\Sigma$ and $H$, where $\Sigma$ is the auxiliary Higgs with a large quartic and $H$ is the MSSM Higgs field. The potential is

$$
V=m_{H}^{2}|H|^{2}+m_{\Sigma}^{2}|\Sigma|^{2}-\kappa^{2}\left(\Sigma^{\dagger} H+\text { h.c. }\right)+\lambda_{\Sigma}|\Sigma|^{4} .
$$

We first consider the decoupling limit where one linear combination of $H$ and $\Sigma$ has a large positive mass-squared. In this limit the light mass eigenstate is

$$
H_{1}=\sin \gamma H+\cos \gamma \Sigma
$$

where $\gamma$ is the mixing angle that diagonalizes the quadratic terms. The effective potential is then

$$
V_{\text {eff }}=m_{1}^{2}\left|H_{1}\right|^{2}+\lambda_{\Sigma} \cos ^{4} \gamma\left|H_{1}\right|^{4}
$$

where $m_{1}^{2}$ is the light mass-squared eigenvalue. In this limit, the potential has induced a quartic interaction for the light Higgs. In the case where $m_{2}^{2} \gg m_{1}^{2}$, this limit generally requires a tuning proportional to $m_{1}^{2} / m_{2}^{2}$, but we do not need such an extreme hierarchy in a realistic theory.

The other limit we are interested in occurs when $\lambda_{\Sigma}$ is large and $\kappa^{2}$ is treated as a perturbation. We focus on the CP-even Higgs bosons and write

$$
\Sigma=\frac{1}{\sqrt{2}}\left(\begin{array}{l}
0 \\
\sigma
\end{array}\right), \quad H=\frac{1}{\sqrt{2}}\left(\begin{array}{l}
0 \\
h
\end{array}\right) .
$$

For $\kappa^{2}=0, \Sigma$ and $H$ decouple, and we have

$$
\langle\sigma\rangle=f, \quad f^{2}=-\frac{m_{\Sigma}^{2}}{\lambda_{\Sigma}} .
$$


The heavy mass eigenstate has mass $m_{\sigma}^{2}=2 \lambda_{\Sigma} f^{2}$. If this mass is sufficiently heavy, we can integrate it out to get an effective Higgs potential

$$
V_{\mathrm{eff}}=\frac{1}{2} m_{H}^{2} h^{2}-\kappa^{2} f h+O\left(\kappa^{4}\right)
$$

Note that a tadpole for $h$ has been generated. If $m_{H}^{2}>0$, this gives a stable VEV for $h$,

$$
v_{h}=\langle h\rangle=\frac{\kappa^{2} f}{m_{H}^{2}} .
$$

We see that electroweak symmetry breaking is dominated by a tadpole induced by the heavy Higgs.

Let us understand the approximation in the induced tadpole limit more systematically. Including higher order terms, the potential obtained by integrating out $\Sigma$ has the form

$$
V_{\mathrm{eff}} \sim m_{H}^{2} h^{2}-\kappa^{2} f h\left[1+\frac{\kappa^{2}}{\lambda_{\Sigma} f^{3}} h+\left(\frac{\kappa^{2}}{\lambda_{\Sigma} f^{3}} h\right)^{2}+\cdots\right] .
$$

From this we see that the higher-order terms can be neglected if $\epsilon \ll 1$, where

$$
\epsilon=\frac{\kappa^{2} v_{h}}{\lambda_{\Sigma} f^{3}} \sim \frac{m_{h}^{2}}{m_{\sigma}^{2}} \frac{v_{h}^{2}}{f^{2}} .
$$

In the last step we used $m_{h}^{2} \sim m_{H}^{2}, m_{\sigma}^{2} \sim \lambda_{\Sigma} f^{2}$, and Eq. (1.7). We see that for $f \sim v_{h}$ the expansion is valid when $m_{\sigma} \gg m_{h}$. Since $m_{\sigma}^{2} \sim \lambda_{\Sigma} f^{2}$, this requires a relatively large quartic interaction for $\Sigma$.

A tadpole interaction violates electroweak gauge symmetry explicitly, and might thus be cause for some suspicion. However, the point is that such an interaction is allowed because the VEV of the heavy Higgs breaks electroweak gauge symmetry. In the low energy effective theory, electroweak gauge symmetry is nonlinearly realized by Nambu-Goldstone bosons. For small $\kappa^{2}$, the Nambu-Goldstone fields are contained in $\Sigma$ :

$$
\Sigma=\frac{1}{\sqrt{2}} e^{i \Pi / f}\left(\begin{array}{l}
0 \\
f
\end{array}\right)+\cdots
$$

where $\Pi=\frac{1}{2} \tau_{a} \Pi_{a}(x)$. This transforms correctly under electroweak gauge transformations provided that $\Pi$ transforms nonlinearly in the standard way. The fields $\Pi$ are light, and the effective potential for $H$ can then be written as

$$
V_{\text {eff }}=m_{H}^{2}|H|^{2}-\frac{\kappa^{2} f}{\sqrt{2}}\left[\left(\begin{array}{l}
0 \\
f
\end{array}\right)^{T} e^{-i \Pi / f} H+\text { h.c. }\right]+\cdots \text {. }
$$


The nonlinear transformation of the $\Pi$ fields ensures that the tadpole term is formally invariant under electroweak gauge symmetry. The П-dependent terms in Eq. (1.11) contain important mixing terms between the CP-odd fields, but they do not change the results above for the CP-even fields. It is straightforward to check that the results above reproduce the results of minimizing the full potential.

Even away from the limits discussed above, the VEV of $H$ in our simplified model can be thought of as being 'induced' by $\Sigma$, in the following sense. The mass matrix of the CP-even fields $\sigma$ and $h$ is

$$
\mathcal{M}^{2}=\left(\begin{array}{cc}
2 \lambda_{\Sigma} f^{2}+m_{H}^{2} v_{h}^{2} / f^{2} & -m_{H}^{2} v_{h} / f \\
-m_{H}^{2} v_{h} / f & m_{H}^{2}
\end{array}\right) .
$$

where we have eliminated $m_{\Sigma}^{2}$ and $\kappa^{2}$ in favor of $v_{h}$ and $f$ using the potential minimization conditions. We have $\operatorname{det}\left(\mathcal{M}^{2}\right)=2 \lambda_{\Sigma} f^{2} m_{H}^{2}$, so vacuum stability requires $m_{H}^{2}>0$. Thus, electroweak symmetry would be unbroken if the $\Sigma$ fields were not present.

In the body of the paper we will consider the phenomenology of induced EWSB in detail. We will find that the simplified model presented above gives a good description of the underlying physics of realistic cases, and that roughly half of the phenomenologically allowed parameter space can be thought of as having an induced quartic, and half an induced tadpole. Note that in the decoupling limit, all Higgs couplings approach that of the standard model, and are therefore consistent with observations. However, significant deviations from this limit are allowed by the present data. If we get too close to the decoupling limit the model becomes tuned, but the existence of this limit makes it clear why there is a region where the Higgs has SM-like couplings. This is conceptually similar to models of Higgs as a Nambu-Goldstone boson, where a similar tuned limit determines the phenomenology 3741 .

The induced EWSB mechanism allows the construction of simple SUSY models that explain the observed $125 \mathrm{GeV}$ Higgs without fine-tuning. These models are compatible with gauge coupling constant unification in the simplest possible way, namely that all new fields come in complete $S U(5)$ muliplets. The models are also naturally compatible with precision electroweak constraints.

The simplest $D$-term model is in fact a minor extension of the 'sister Higgs' model described in Ref. [42], where the auxiliary Higgs are the same as sister Higgs fields. However, we focus on a very different regime of parameters where the new $S U(2)_{S}$ gauge group is broken at a scale $\sim 3 \mathrm{TeV}$, naturally suppressing the corrections to precision electroweak observables as well as gauge coupling unification. Ref. [42] instead considered a regime where this breaking scale is low and focused on the effects 
of a large $F$-term generating the quartic $|\Sigma H|^{2}$. In this regime, there is tension between naturalness and precision electroweak constraints, as we will explain below.

The paper is organized as follows. In \&2 we study further the simplified model of induced EWSB introduced above. In \$3 we discuss models where the auxiliary Higgs quartic arises from the $D$-term of a new non-Abelian gauge group. In $\$ 4$ we discuss models where the auxiliary Higgs quartic arises from an F-term and consider a 'hybrid' model involving both $F$ and $D$ terms. Our conclusions are in \$5. Certain details of the behavior of these models under the renormalization group are reserved for an appendix.

\section{A Simplified Model}

We now discuss in more detail the simplified model of EWSB defined in the introduction above. We remind the reader that this model consists of two doublets $H$ and $\Sigma$, with a potential given in Eq. (1.1). The fully realistic models that we discuss later will have additional Higgs fields at low energies, but we will see that many aspects of the models are similar to the simplified model considered here.

This model has 4 parameters. Fixing the scale of electroweak symmetry breaking at $v=246 \mathrm{GeV}$ and the mass of the lightest CP-even Higgs boson $m_{h}=125 \mathrm{GeV}$ we have 2 remaining parameters, which we take to be the quartic coupling $\lambda_{\Sigma}$ and the VEV $f$ of the auxiliary Higgs field

$$
\langle\Sigma\rangle=\frac{1}{\sqrt{2}}\left(\begin{array}{l}
0 \\
f
\end{array}\right)
$$

The qualitative features of this parameter space can be understood from the two limits of this model discussed in the introduction, and this is what we turn to next.

\subsection{Decoupling Limit}

In the decoupling limit, one linear combination of Higgs fields has a large positive mass-squared term and therefore no VEV. We can analyze this limit by diagonalizing the quadratic terms in the potential Eq. (1.1) by writing

$$
\left(\begin{array}{c}
H \\
\Sigma
\end{array}\right)=\left(\begin{array}{cc}
s_{\gamma} & c_{\gamma} \\
c_{\gamma} & -s_{\gamma}
\end{array}\right)\left(\begin{array}{l}
H_{1} \\
H_{2}
\end{array}\right),
$$

where $s_{\gamma}=\sin \gamma, c_{\gamma}=\cos \gamma$. The potential in terms of these fields is

$$
V=m_{1}^{2}\left|H_{1}\right|^{2}+m_{2}^{2}\left|H_{2}\right|^{2}+\lambda_{\Sigma} c_{\gamma}^{4}\left|H_{1}\right|^{4}+\cdots
$$


where we assume $m_{2}^{2} \gg\left|m_{1}^{2}\right|$ is the large positive mass-squared eigenvalue. Integrating out $H_{2}$, we then obtain the effective potential for the light Higgs doublet $H_{1}$. The light CP-even Higgs mass is therefore

$$
m_{h}^{2}=2 \lambda_{\Sigma} c_{\gamma}^{4} v^{2}
$$

The mixing angle $\gamma$ is determined by the VEVs from the requirement that $H_{2}$ have vanishing $\mathrm{VEV}$ :

$$
\tan \gamma=\frac{v_{h}}{f}
$$

so we have

$$
\lambda_{\Sigma}=\frac{m_{h}^{2} v^{2}}{2 f^{4}} .
$$

This explains the minimum value of $\lambda_{\Sigma}$ in Fig. 3. The decoupling limit is approached as $\lambda_{\Sigma}$ approaches the value Eq. (2.6) from above. In this limit the soft masses $m_{H}^{2}$ and $m_{\Sigma}^{2}$ both grow arbitrarily large as is shown in Fig. 2, though with a ratio that allows one eigenstate to remain light. Eq. 2.6 in fact represents a phenomenological lower bound on the quartic: for values just below the decoupling value the Higgs soft mass Eq. 2.9 changes sign and the vacuum is consequently destabilized, while for very small values of the quartic the Higgs becomes the heavier eigenstate of the mass matrix, Eq. 1.12), indicating the presence of scalars lighter than the $125 \mathrm{GeV}$ Higgs.

In the decoupling limit, the effective theory of electroweak symmetry breaking is that of a single Higgs doublet, and it is clear that all Higgs couplings approach those of the standard model. Because the current data favors a SM-like Higgs, this limit is compatible with the Higgs data. Approaching this limit asymptotically, the model becomes fine-tuned because the heavy mass eigenstate contributes to the mass of the light mass eigenstate:

$$
\Delta m_{1}^{2} \sim \frac{\lambda_{\Sigma} s_{\gamma}^{2} c_{\gamma}^{2}}{16 \pi^{2}} m_{2}^{2}
$$

However, the fine-tuning decreases rapidly away from this limit and the experimental constraints on the Higgs couplings do not require the model to be tuned. This will be made quantitative below. However, this limit makes it clear that the model can approximate the standard model, and therefore account for the current data which are consistent with a SM Higgs. 


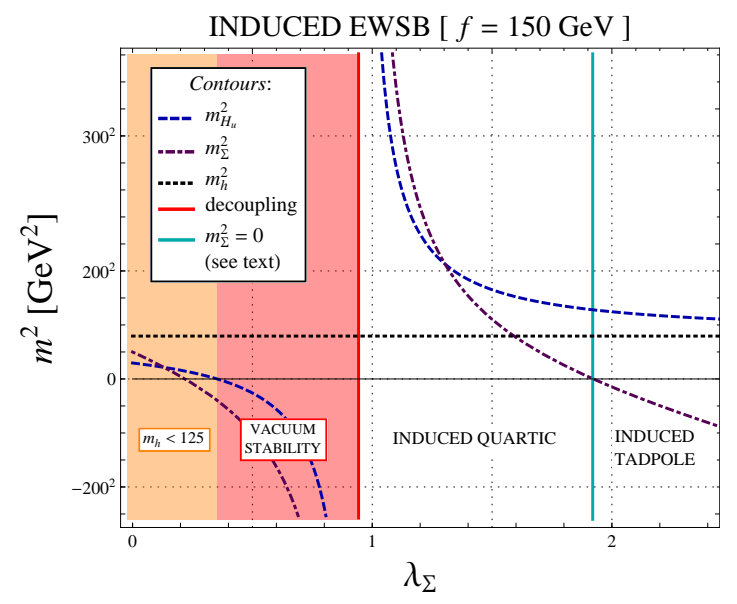

Fig. 2. Higgs mass parameters as a function of the auxiliary Higgs quartic $\lambda_{\Sigma}$ for $f=$ $150 \mathrm{GeV}$. The decoupling limit occurs near $\lambda_{\Sigma}=1 . m_{\Sigma}^{2}$ crosses 0 near $\lambda_{\Sigma}=2$, indicating a clear transition between induced quartic and induced tadpole regimes.
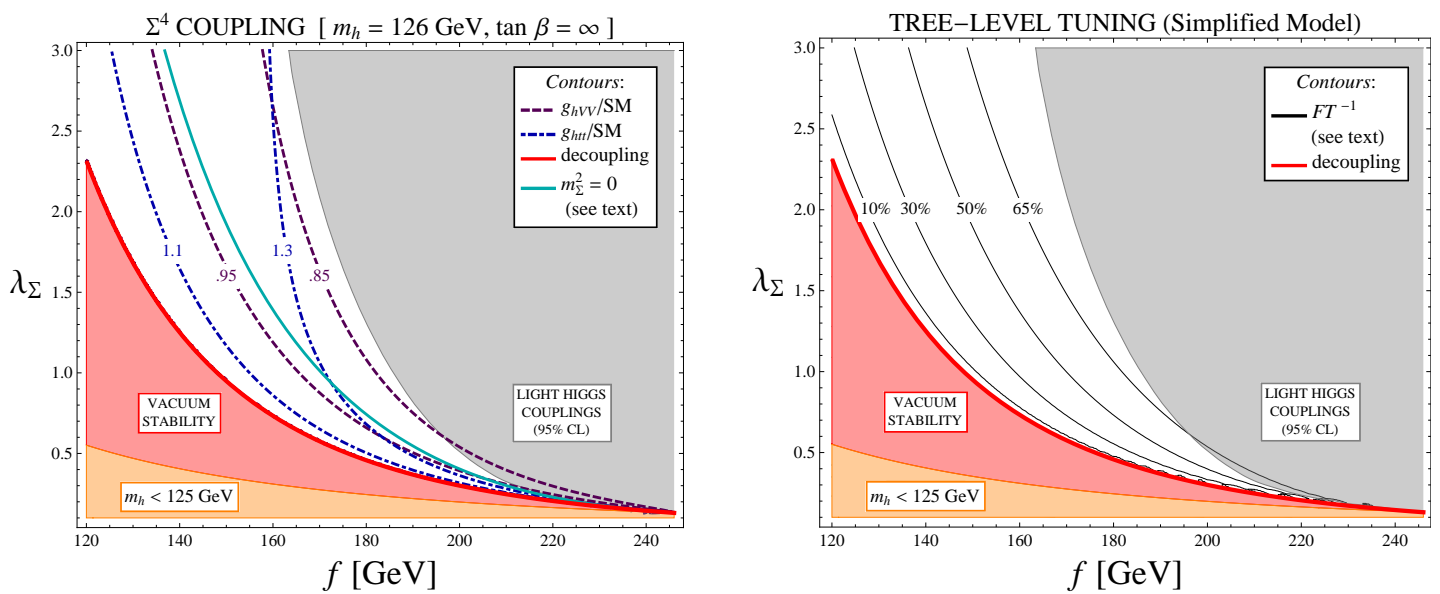

Fig. 3. Parameter space of the simplified two Higgs doublet model describing the mechanism of induced EWSB. Left: Couplings of light Higgs couplings to weak gauge bosons and top quarks. Right: Tree-level tuning of the simplified model, as described in text. 


\subsection{Higgs Phenomenology}

The results above allow us to easily understand the phenomenologically allowed parameter range of this model shown in Fig. 3. The red and gold regions indicate regions of vacuum instability and light scalars, respectively. The grey region is excluded by the measured Higgs couplings at 95\% confidence level. For large values of $f$, significant Higgs mixing is unavoidable and the model becomes incompatible with measured Higgs couplings.

The red boundary represents the decoupling limit $m_{\Sigma}^{2} \rightarrow \infty$ where all couplings are SM-like. We emphasize that the light Higgs can still have a finite admixture of the $\Sigma$ fields in this limit. This should be contrasted to models like the NMSSM where the Higgs gets a quartic by mixing with a singlet; in these models, large mixing necessarily implies suppression of the Higgs couplings to gauge bosons and fermions which is disfavored by LHC and LEP data.

The model becomes fine-tuned as we approach the decoupling limit, but we do not need to be very close to this limit to satisfy the phenomenological constraints. We illustrate this in right panel of Fig. 3 by plotting a standard measure of tuning [43], namely the inverse of the function

$$
\mathrm{FT}=\max \left(\frac{\partial \log \left\{v, f, m_{h}\right\}}{\partial \log m_{\text {input }}^{2}}\right),
$$

where $m_{\text {input }}$ stands for any of the dimensionful parameters defining the potential of Eq. (1.1). Explicitly the relevant relations are

$$
\begin{aligned}
m_{H}^{2} & =m_{h}^{2}\left(1+\frac{m_{h}^{2} v_{h}^{2}}{2 \lambda_{\Sigma} f^{4}-m_{h}^{2} v^{2}}\right) \\
m_{\Sigma}^{2} & =m_{h}^{2} v_{h}^{2} \frac{2 \lambda_{\Sigma} f^{2}-m_{h}^{2}}{2 \lambda_{\Sigma} f^{4}-m_{h}^{2} v^{2}}-\lambda_{\Sigma} f^{2} \\
\kappa^{2} & =m_{h}^{2} v_{h} f \frac{2 \lambda_{\Sigma} f^{2}-m_{h}^{2}}{2 \lambda_{\Sigma} f^{4}-m_{h}^{2} v^{2}} .
\end{aligned}
$$

These become singular in the decoupling limit $2 \lambda_{\Sigma} f^{4} \rightarrow m_{h}^{2} v^{2}$, accounting for the large tuning there. When we construct explicit models, there will be other potential sources of tuning arising from loops involving heavier superpartners, and we will include these as well.

As we move away from the minimum value of $\lambda_{\Sigma}$, the mass of the heavy CP-even mass eigenstate first decreases, but then begins to increase as we enter the regime where the VEV of the light Higgs field is induced by an effective tadpole. Because we 
do not want to tune near the decoupling limit, and we do not want to take $\lambda_{\Sigma}$ nonperturbatively large, we are not really close to either limit. It is nonetheless interesting to ask whether we are 'closer' to one limit or the other for phenomenologically allowed parameters.

There is no sharp boundary between the two regimes. The decoupling limit requires $m_{\Sigma}^{2} \gg 0$, while the induced tadpole limit requires $m_{\Sigma}^{2}<0$. A useful definition of the boundary is thus where $m_{\Sigma}^{2}=0$ : as can be seen in Fig. 2, the Higgs soft mass beyond this point nearly coincides with its asymptotic value $m_{H}^{2}=m_{h}^{2}$ indicating a strongly induced tadpole. We illustrate this also in Fig. 3, and there we see that in roughly half of the allowed parameter space an induced tadpole is playing an important role. In these regions, a physical characteristic of interest is that the Higgs quartic coupling is drastically suppressed relative to its SM value by powers of the small parameter $\epsilon$ (see Eqs. (1.8) and (1.9)). The simplified model omits the tree-level contribution to the quartic from the $S U(2)_{W} \times U(1)_{Y} D$-terms, so the quartic in the simplified model can be viewed as a correction to the tree-level mass. The quartic coupling, as a 'smoking gun' of the induced tadpole, will be subject to experimental scrutiny only with significantly more Higgs data than is presently available, but we find it illustrative to emphasize this feature in the models we describe below. Thus in realistic cases, we will find it convenient to indicate the importance of the induced tadpole by focussing on regions of the parameter space where the Higgs quartic coupling is smaller than a benchmark value which we will take to be its tree-level value in the MSSM (corresponding to approximately half of the SM value).

We now proceed to a discussion of two separate complete models that can realize the mechanism of induced EWSB with perturbative dynamics.

\section{A D-term Model}

In this section we consider the case where the large quartic for the auxiliary Higgs fields arises from the $D$-terms of a new gauge interaction. We will see that the simplest versions of this model have a phenomenology very similar to the simplified model discussed in $\$ 2$. New $D$ terms have been previously considered as a way to generate large quartics for the Higgs by embedding the Higgs into a multiplet charged under the new gauge group [22,23]. However, these models do not preserve gauge coupling unification without significant additional structure because the Higgs fields are not part of a complete $S U(5)$ multiplet. On the other hand, we preserve gauge coupling unification in our approach simply by taking the auxiliary Higgs fields to be part of a complete $S U(5)$ multiplet. 


\subsection{The Model}

We assume the existence of a new gauge interaction with gauge group $S U(2)_{S}$, with additional matter fields given in Table 1. Color triplets $T$ and $\bar{T}$ are included so

\begin{tabular}{c|c|ccc} 
& $S U(2)_{S}$ & $S U(3)_{C}$ & $S U(2)_{W}$ & $U(1)_{Y}$ \\
\hline$\Phi$ & $\square$ & 1 & 1 & 0 \\
$\bar{\Phi}$ & $\square$ & 1 & 1 & 0 \\
$\Sigma_{u}$ & $\square$ & 1 & $\square$ & $\frac{1}{2}$ \\
$\Sigma_{d}$ & $\square$ & 1 & $\square$ & $-\frac{1}{2}$ \\
$T$ & $\square$ & $\square$ & 1 & $-\frac{2}{3}$ \\
$\bar{T}$ & $\square$ & $\square$ & 1 & $\frac{2}{3}$ \\
\hline
\end{tabular}

Table 1. Field content of the $D$-term model.

that the model consists of complete $S U(5)$ multiplets, and therefore preserves gauge coupling unification. There are 6 'flavors' of $S U(2)_{S}$, and therefore it has vanishing 1-loop beta function. This naturally allows a large range of $S U(2)_{S}$ gauge coupling constants at the weak scale. We will discuss the RG behavior in more detail below.

The $S U(2)_{S}$ gauge symmetry will be broken at the weak scale by VEVs of the fields $\Phi$ and $\bar{\Phi}$, as well as the auxiliary Higgs fields $\Sigma_{u, d}$. The $\Sigma_{u, d}$ VEVs break custodial symmetry, and therefore contribute to the electroweak $T$ parameter. We will see below that in order for this to be sufficiently small, we will need $u \sim\langle\Phi\rangle,\langle\bar{\Phi}\rangle \gg f \sim\left\langle\Sigma_{u, d}\right\rangle$. Because we require $f \sim 100 \mathrm{GeV}$, we must have $u \gtrsim \mathrm{TeV}$ to satisfy precision electroweak constraints. (This also suppresses the mixing between $S U(2)_{W}$ and $S U(2)_{S}$ that would otherwise ruin the unification prediction of the gauge couplings.) This creates a potential tuning problem because the $S U(2)_{S} D$-term quartic generally gives a tree-level contribution to the $\Sigma$ quadratic of order $g_{S}^{2} u^{2} \sim \mathrm{TeV}^{2}$. We can avoid this problem by assuming that $S U(2)_{S}$ is broken along an approximately $D$-flat direction. 1 We are thus led to introduce the superpotential

$$
\Delta W=\lambda_{\Phi} S\left(\Phi \bar{\Phi}-w^{2}\right)
$$

and to impose a $Z_{2}$ symmetry ensuring approximately equal soft masses for $\Phi$ and $\bar{\Phi}$. The potential for these fields then has the form

$$
V_{\Phi}=m_{\Phi}^{2}\left(\Phi^{\dagger} \Phi+\bar{\Phi}^{\dagger} \bar{\Phi}\right)+\lambda_{\Phi}^{2}\left(\Phi \bar{\Phi}+w^{2}\right)^{2}+B_{\Phi}(\Phi \bar{\Phi}+\text { h.c. })+V_{D}
$$

\footnotetext{
${ }^{1}$ This mechanism is also used in models where the Higgs gets a contribution to its quartic from new $D$-terms 22,23 .
} 
with $V_{D}$ the potential arising from $D$ terms of $S U(2)_{S}$. A nonzero VEV is then established provided $B_{\Phi}>m_{\Phi}^{2}+\lambda^{2} w^{2}$ :

$$
\langle\Phi\rangle=\frac{1}{\sqrt{2}}\left(\begin{array}{l}
0 \\
u
\end{array}\right), \quad\langle\bar{\Phi}\rangle=\frac{1}{\sqrt{2}}\left(\begin{array}{l}
\tilde{u} \\
0
\end{array}\right),
$$

with

$$
u=\tilde{u}= \pm \sqrt{\frac{2 B_{\Phi}-2\left(m_{\Phi}^{2}+\lambda_{\Phi}^{2} w^{2}\right)}{\lambda_{\Phi}^{2}}} .
$$

The quartic for $\Sigma$ induced from $S U(2)_{S} D$-terms vanishes in the SUSY limit, so we must have $m_{\Phi}^{2} \sim g_{S}^{2} u^{2}$ to avoid a cancellation. Integrating out the scalar fields in $\Phi$ and $\bar{\Phi}$ gives the quartic

$$
\lambda_{\Sigma} \simeq \frac{g_{S}^{2}}{8}\left(1+\frac{g_{S}^{2}\left(u^{2}+\tilde{u}^{2}\right)}{8 m_{\Phi}^{2}}\right)^{-1} .
$$

This requires $\lambda_{\Phi} \sim g_{S}$, as can be seen from Eq. (3.4). This mechanism therefore requires the existence of two new order-1 dimensionless couplings at the weak scale $\left(g_{S}\right.$ and $\left.\lambda_{\Phi}\right)$. This raises the issue of Landau poles and perturbative unification, which will be discussed below.

\subsection{A Simple Low-energy Limit}

This model can have up to 6 Higgs doublets, 2 from the MSSM fields $H_{u, d}$ and 4 more from $\Sigma_{u, d}$. To illustrate the phenomenology, we will focus on a simple limit where only one of the new Higgs doublets gets a VEV. The effective theory below the $S U(2)_{S}$ breaking scale is then a 3-Higgs doublet model. Specifically, we assume that

$$
\left\langle\Sigma_{d}\right\rangle=\frac{1}{\sqrt{2}}\left(\begin{array}{ll}
0 & 0 \\
0 & f
\end{array}\right), \quad\left\langle\Sigma_{u}\right\rangle=0 .
$$

For this limit to be exact requires that ' $B \mu$ terms' of the form $\Sigma_{u} \Sigma_{d}$ and $\Sigma_{u} H_{d}$ vanish, which is not natural. However, a mild hierarchy among the SUSY breaking terms can easily make the simplified limit we study a good first approximation. In any case, the parameter space of the full model is too large to study, and we must make some simplifying assumptions to proceed.

With these simplifying assumptions, the only terms that are relevant are those that mix $\Sigma_{d}$ and the MSSM Higgs fields. These are the superpotential terms

$$
\Delta W=\mu H_{u} H_{d}+\lambda_{u} H_{u} \Sigma_{d} \Phi+\bar{\lambda}_{u} H_{u} \Sigma_{d} \bar{\Phi}
$$


and the soft SUSY breaking terms in the Higgs potential

$$
V=m_{H_{u}}^{2}\left|H_{u}\right|^{2}+m_{H_{d}}^{2}\left|H_{d}\right|^{2}+m_{\Sigma_{d}}^{2}\left|\Sigma_{d}\right|^{2}+B \mu H_{u} H_{d}+B_{u} H_{u} \Sigma_{d}
$$

In addition, the theory has the $D$-term quartic from the $S U(2)_{S} \times S U(2)_{W} \times U(1)_{Y}$ gauge interactions.

The couplings $\lambda_{u}$ and $\bar{\lambda}_{u}$ contribute the mass terms

$$
\Delta V=\left(\left|\lambda_{u}\right|^{2}+\left|\bar{\lambda}_{u}\right|^{2}\right) u^{2}\left(\left|H_{u}\right|^{2}+\left|\Sigma_{d}\right|^{2}\right)
$$

Precision electroweak tests and unification require $u \gtrsim \mathrm{TeV}$, so in order for these mass terms not to be unnaturally large, we must take $\lambda_{u}, \bar{\lambda}_{u} \lesssim v / u \sim 0.1$. This is sufficiently small that $\lambda_{u}, \bar{\lambda}_{u}$ do not contribute significantly to the quartic. Ref. [42] analyzed exactly the same model, focussing on the Higgs quartic generated by $\lambda_{u}$. However, as discussed here this is fine-tuned at the percent level if $u \sim \mathrm{TeV}$. Ref. [42] uses a less stringent bound on the $T$ parameter than we do, allowing smaller values of $u$.

The couplings $\lambda_{u}, \bar{\lambda}_{u}$ are however relevant because we require $B_{u} \sim(100 \mathrm{GeV})^{2}$, and we expect $B_{u}$ to be $\lambda_{u} u$ times a SUSY breaking mass. This is consistent with taking $\lambda_{u}, \bar{\lambda}_{u} \sim 0.1$, which we will assume in the following. We now define

$$
\tan \beta=\frac{v_{u}}{v_{d}}, \quad \tan \gamma=\frac{v_{h}}{f}, \quad v_{h}=\sqrt{v_{u}^{2}+v_{d}^{2}} .
$$

The minimization conditions are as follows, dropping the now unnecessary subscript from $\Sigma_{d}$ :

$$
\begin{aligned}
& m_{H_{d}}^{2}=B \mu \tan \beta-\frac{1}{2} m_{Z}^{2}\left(s_{\gamma}^{2} \cos 2 \beta+c_{\gamma}^{2}\right) \\
& m_{H_{u}}^{2}=B \mu \cot \beta+B_{u} \frac{\cot \gamma}{s_{\beta}}+\frac{1}{2} m_{Z}^{2}\left(s_{\gamma}^{2} \cos 2 \beta+c_{\gamma}^{2}\right)-\frac{1}{2} \lambda_{u}^{2} v^{2} c_{\gamma}^{2} \\
& m_{\Sigma}^{2}=B_{u} s_{\beta} \tan \gamma-\frac{1}{2} m_{Z}^{2}\left(s_{\gamma}^{2} \cos 2 \beta+c_{\gamma}^{2}\right)-\frac{1}{2} g_{S}^{2} v^{2} c_{\gamma}^{2}-\frac{1}{2} \lambda_{u}^{2} v^{2} s_{\beta}^{2} s_{\gamma}^{2} .
\end{aligned}
$$

The MSSM formulas are recovered in the limit $s_{\gamma} \rightarrow 1, B_{u} \rightarrow 0$. In practice, we solve these for $\left(B \mu, B_{u}, m_{\Sigma}^{2}\right)$ respectively.

The $S U(2)_{W} \times U(1)_{Y} D$-term quartics necessarily give a small contribution to a $125 \mathrm{GeV}$ Higgs mass, so it is a good approximation to neglect them. We also assume that stop loops do not contribute a large quartic. In our final numerical results we include these effects, but we can obtain a good analytic approximation by dropping them. In this approximation, the mass matrix for the $C P$-even neutral Higgs bosons 
is

$$
\mathcal{M}^{2}=\left(\begin{array}{ccc}
m_{H_{u}}^{2} & -\frac{v_{d}}{v_{u}} m_{H_{d}}^{2} & -\frac{1}{v_{u} f}\left(m_{H_{u}}^{2} v_{u}^{2}-m_{H_{d}}^{2} v_{d}^{2}\right) \\
-\frac{v_{d}}{v_{u}} m_{H_{d}}^{2} & m_{H_{d}}^{2} & 0 \\
-\frac{1}{v_{u} f}\left(m_{H_{u}}^{2} v_{u}^{2}-m_{H_{d}}^{2} v_{d}^{2}\right) & 0 & \frac{1}{f^{2}}\left(m_{H_{u}}^{2} v_{u}^{2}-m_{H_{d}}^{2} v_{d}^{2}\right)+\frac{1}{4} g_{S}^{2} f^{2}
\end{array}\right) .
$$

We have set $\lambda_{u}, \bar{\lambda}_{u} \simeq 0$ as explained above. Assuming large $\tan \beta\left(v_{d} \ll v_{u}, f\right)$ we obtain an illustrative upper bound on the lowest eigenvalue:

$$
m_{h}^{2}<m_{Z}^{2} \cos ^{2} 2 \gamma+\frac{1}{4} g_{S}^{2} v^{2} \cos ^{4} \gamma
$$

This result is similar to what is obtained in models where new $D$-terms modify the Higgs quartic, but in this case the effect comes from contributions to the auxiliary Higgs quartic while allowing the physical Higgs quartic to remain suppressed.

Below the $S U(2)_{S}$ breaking scale, this simplified limit has 6 parameters:

$$
m_{H_{u}}^{2}, m_{H_{d}}^{2}, B \mu, m_{\Sigma_{d}}^{2}, B_{u}, \lambda_{\Sigma}
$$

We write

$$
\lambda_{\Sigma}=\frac{g_{S \mathrm{eff}}^{2}}{8}
$$

where $g_{S \text { eff }}$ and the $S U(2)_{S}$ gauge coupling coincide in the limit $m_{\Phi}^{2} \rightarrow \infty$ (see Eq. (3.5p). After fixing $v$ and the mass of the lightest $C P$ even mass eigenstate, there are 4 free parameters. We take two of these to be $f$ and $g_{S \text { eff }}$, and scan over the rest. We will see that the parameter space in the plane of $f$ and $g_{S \text { eff }}$ reproduces the main features of the simplified model discussed in \$2. In particular, the lower bound on $g_{S \text { eff }}$ corresponds to the limit where one linear combination of Higgs doublets decouples, and this line is determined to a good approximation by Eq. (2.6) for the simplified model.

The couplings of the light Higgs to the $W / Z$, the top quark, and the bottom quark relative to their SM values are given by

$$
\begin{aligned}
c_{V} & =\left\langle\Sigma_{d} \mid h\right\rangle \cos \gamma+\left(\left\langle H_{u}^{0} \mid h\right\rangle \sin \beta+\left\langle H_{d} \mid h\right\rangle \cos \beta\right) \sin \gamma \\
c_{t} & =\frac{\left\langle H_{u}^{0} \mid h\right\rangle}{\sin \gamma \sin \beta} \\
c_{b} & =\frac{\left\langle H_{d}^{0} \mid h\right\rangle}{\sin \gamma \cos \beta} .
\end{aligned}
$$

We show the allowed region for a benchmark case $\tan \beta=5$ in Fig. 4, now including the subleading SM quartic contributions. As in the simplified model, the lower 
boundary corresponds to the decoupling limit for the auxiliary Higgs bosons, and near this limit the light Higgs is SM-like. The grey region is excluded by Higgs coupling constraints, determined as in [44]. This is the region of small $\sin \gamma$, which suppresses $c_{V}$ and enhances $c_{t}$. Fig. 4 also shows the allowed points where $\lambda_{h}$ is smaller than half of its SM value. In such theories, the Higgs cubic and quartic self-couplings are highly suppressed compared the SM, which can be measurable in future experiments or with large integrated luminosity via enhanced di-Higgs production at the highenergy LHC [45 47]. This also has conceptual importance, since it corresponds to the regime where an induced tadpole is important, as discussed in \$2. The tree-level MSSM gives a quartic that is about half of the SM value (for large tan $\beta$ ), so these points are also very far from the usual SUSY solutions, all of which strive to obtain a Higgs quartic close to SM value.

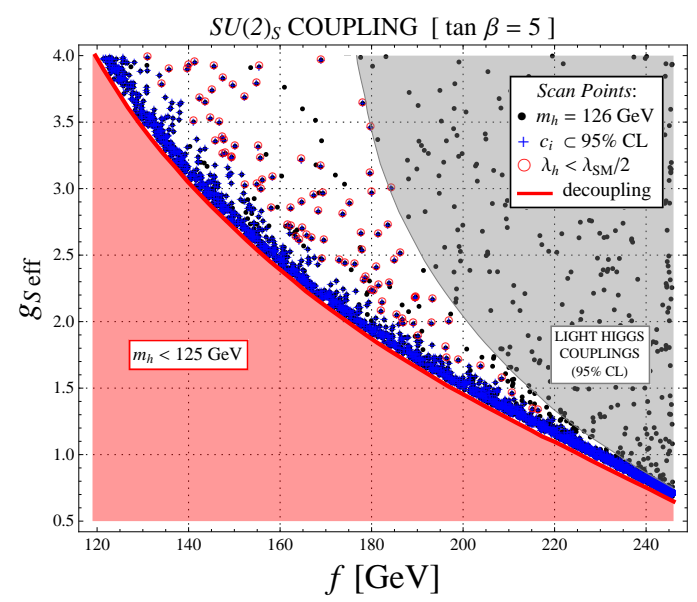

Fig. 4. Scan of the parameter space for a model where auxiliary Higgs quartics arise from non-decoupling $D$ terms. All points have $m_{h} \simeq 125 \mathrm{GeV}$. A blue cross is added if the Higgs couplings are compatible with experimental values, and a red circle is added to surviving points if the physical Higgs has a quartic of order half its SM value, indicating regions where the induced tadpole is important.

\subsection{Electroweak Precision Tests}

We turn now to the issue of corrections to electroweak precision tests. The strongest constraints are oblique radiative corrections, which we treat using the formalism of [48, 49.

There are two main effects. The first comes from the $S U(2)_{S}$ breaking threshold at the scale $g_{S} u$. The VEVs of the auxiliary Higgs fields break both $S U(2)_{S}$ and the 
electroweak group. This breaks custodial symmetry, and therefore gives a positive tree-level contribution to the $T$-parameter. The other main effect comes from loops of the auxiliary Higgs fields. As we explain below, for large $g_{S}$ the auxiliary Higgs fields contribute positively to the $S$ parameter like a heavy Higgs doublet, while giving a negligible contribution to the $T$-parameter.

These two effects partially offset each other, in the sense that a positive $S$ parameter allows a larger positive $T$ parameter, allowing lower values of the $S U(2)_{S}$ breaking scale than would be allowed if only one of these effects was present. This in turn reduces the leading source of fine-tuning in this model.

$S U(2)_{S}$ breaking: The $S U(2)_{S}$ gauge bosons mix with the electroweak gauge bosons due to VEVs of the auxiliary Higgs bosons. A $T$-parameter arises because the $W_{S}^{1,2}$ gauge bosons do not mix with the $W^{ \pm}$, while the $W_{S}^{3}$ does mix with the neutral SM gauge bosons.

The mixing of the neutral gauge bosons arises from the VEVs of the axiliary Higgs fields via the kinetic term. For illustration we will focus on the case of a single auxiliary Higgs, but the generalization to additional Higgs is straightforward. We have

$$
\Delta \mathcal{L}=\left|-i g \frac{\tau_{3}}{2} W_{\mu}^{3} \Sigma_{d}+i g^{\prime} B_{\mu} \frac{1}{2} \Sigma_{d}-i g_{S} W_{S \mu}^{3} \Sigma_{d} \tau_{3}\right|^{2}
$$

and a mass matrix for the neutral gauge bosons in the basis $\left(W_{\mu}^{3}, B_{\mu}, W_{S \mu}^{3}\right)$ given by

$$
\mathcal{M}^{2}=\frac{1}{4}\left(\begin{array}{ccc}
g^{2} v^{2} & -g g^{\prime} v^{2} & -g g_{S} f^{2} \\
-g g^{\prime} v^{2} & g^{\prime 2} v^{2} & g^{\prime} g_{S} f^{2} \\
-g g_{S} f^{2} & g^{\prime} g_{S} f^{2} & g_{S}^{2}\left(f^{2}+u^{2}+\tilde{u}^{2}\right)
\end{array}\right) \text {. }
$$

Integrating out the heavy neutral gauge boson, the leading correction to the effective theory is given by

$$
\Delta \mathcal{L}_{\text {eff }}=-\frac{1}{8} \frac{\left(g^{2}+g^{\prime 2}\right) f^{4}}{u^{2}+\tilde{u}^{2}} Z^{\mu} Z_{\mu}
$$

where $Z_{\mu}=\left(g W_{\mu}^{3}-g^{\prime} B_{\mu}\right) / \sqrt{g^{2}+g^{\prime 2}}$. This is a correction to the $Z$ mass without a corresponding correction to the $W$ mass, which gives

$$
\Delta T=-\frac{1}{\alpha} \frac{\delta m_{Z}^{2}}{m_{Z}^{2}}=\frac{1}{\alpha} \frac{f^{4}}{\left(u^{2}+\tilde{u}^{2}\right) v^{2}} .
$$

The leading order contribution to the $S$ parameter comes from mixing between $W_{\mu}^{3}$ and $B_{\mu}$ generated by mixing with an intermediate $S U(2)_{S}$ boson $W_{S \mu}^{3}$. From the 


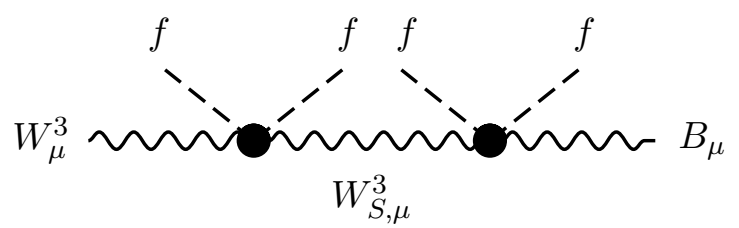

Fig. 5. Tree-level diagram contributing to the $S$ parameter.

tree-level diagram in Fig. 5 we can integrate out $W_{S}^{3}$ with $m_{W_{S}^{3}}^{2}=g_{S}^{2}\left(u^{2}+\tilde{u}^{2}+f^{2}\right) / 4$ to arrive at the form factor

$$
\begin{aligned}
\Pi_{3 B}\left(p^{2}\right) & =-\frac{\left(g g_{S} f^{2}\right)\left(g^{\prime} g_{S} f^{2}\right)}{4\left(p^{2}-m_{W_{S}^{3}}^{2}\right)} \\
& =\frac{g g^{\prime} f^{4}}{u^{2}+\tilde{u}^{2}+f^{2}}\left[1+\frac{4 p^{2}}{g_{S}^{2}\left(u^{2}+\tilde{u}^{2}+f^{2}\right)}+\mathcal{O}\left(p^{4}\right)\right] .
\end{aligned}
$$

Thus for the $S$ parameter,

$$
S=\frac{16 \pi}{g g^{\prime}} \Pi_{3 B}^{\prime}(0) \simeq 64 \pi \frac{f^{4}}{g_{S}^{2}\left(u^{2}+\tilde{u}^{2}+f^{2}\right)^{2}} .
$$

Taking conservative values (see below) $g_{S}=1, \tan \gamma=2$, and $u, \tilde{u} \simeq \mathrm{TeV}$, we find a completely negligible $S$ parameter, $S \simeq 5 \times 10^{-3}$.

Auxiliary Higgs bosons: In the limit where $g_{S}$ is large, the auxiliary Higgs fields act as a heavy electroweak breaking sector, and there is a danger of a large contribution to the $S$ and $T$ parameters. In this limit we can integrate out the auxiliary Higgs fields and write an effective theory where electroweak symmetry is nonlinearly realized. This effective theory will contain explicit $W$ and $Z$ mass terms proportional to $f$. This effective Lagrangian will also contain a 4-derivative operator corresponding to the $S$-parameter. This is dimensionless and hence depends only logarithmically on the mass of the heavy states:

$$
\Delta S \simeq \frac{1}{12 \pi} \ln \frac{m_{\sigma}^{2}}{m_{h}^{2}}
$$

where $m_{\sigma}$ is the mass of the heavy auxiliary Higgs bosons. This expression is correct only in an approximation where the logarithm dominates, but this is the only limit where such a contribution is large. The low-energy effective theory also contains vector boson mass terms that violate custodial symmetry, but these are proportional 


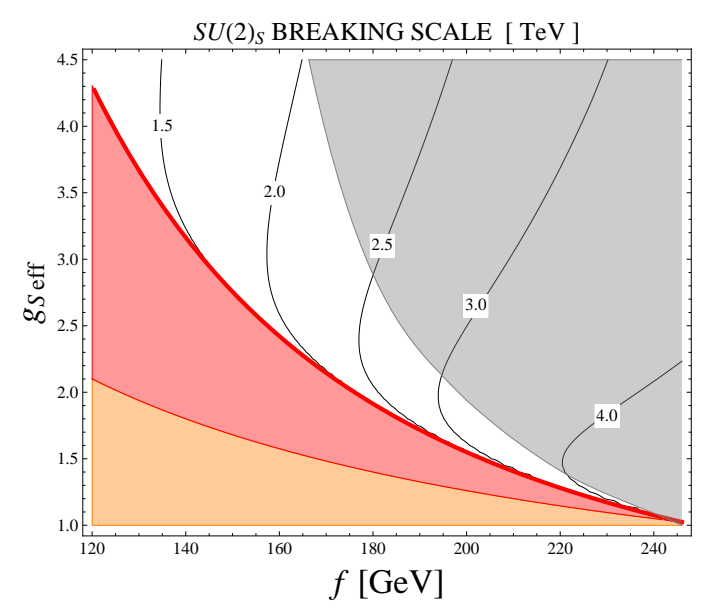

Fig. 6. Bounds on the $S U(2)_{S}$ breaking scale $u$ from precision electroweak constraints.

to $f^{2}$, and hence the $T$-parameter is given by

$$
\Delta T \simeq-\frac{3}{16 \pi c_{W}^{2}} \ln \frac{m_{\sigma}^{2}}{m_{h}^{2}} \times \frac{f^{2}}{v^{2}} .
$$

This gives a small contribution to the $T$-parameter unless $f \simeq v$.

The other limit that is easy to understand is the decoupling limit, which corresponds to taking $g_{S}$ as small as possible. In this limit the auxiliary Higgs fields are again heavy, but their masses are nearly electroweak-preserving. Therefore there is a contribution to the $S$ and $T$ parameter that vanish as we approach this limit. Explicitly for the $S$ parameter, we have

$$
\Delta S=\frac{g_{S}^{2}}{96 m_{\sigma}^{2}} \frac{f^{2} v_{h}^{2}}{v^{2}}
$$

The corrections are therefore negligible in the decoupling limit.

To include these effects, we include the full perturbative contribution from the Higgs sector using the results of [50]. We impose the $95 \%$ confidence level $S-T$ constraint from [51] with $U=0$. We find that the $S$ parameter contribution from the auxiliary Higgs bosons is never so large that it cannot be offset by a positive $T$ contribution. The result can therefore be given as a constraint on the $S U(2)_{S}$ breaking scale $u$, and is shown in Fig. 6. We see that this scale must be at least 2-3 $\mathrm{TeV}$ in most of the parameter space. 


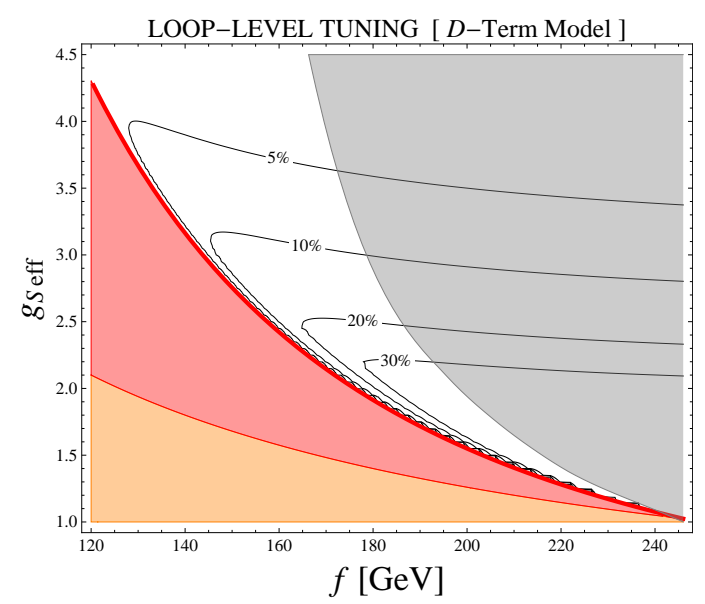

Fig. 7. Fine tuning in the simplified model with non-decoupling $D$ terms. Tree-level contributions dominate near the decoupling (bold red) contour; two-loop contributions from the heavy scalar $\Phi$ dominate at increased values of $f, g_{S}$ where $u, \tilde{u} \gg v$ and $m_{\Phi}^{2} \gg m_{\Sigma}^{2}$. Excluded regions correspond to those described in Fig. 3 .

\subsection{Naturalness}

We now discuss the question of naturalness in this model. We have seen above that the $S U(2)_{S}$ breaking scale is required to be in the $\mathrm{TeV}$ range, while the auxiliary Higgs fields (which are also charged under $S U(2)_{S}$ ) must have VEVs near the $100 \mathrm{GeV}$ scale. Loop corrections can potentially destabilize this little hierarchy and give rise to fine-tuning. From Eq. 3.5 we know that the mass-squared of $\Phi$ must be large, of order $g_{S}^{2} u^{2}$, in order to have an unsuppressed quartic coupling for the $\Sigma$ fields. The only large coupling between the $\Phi$ and $\Sigma$ fields is the $S U(2)_{S}$ gauge coupling, and so the leading correction to the $\Sigma$ mass arises at 2 loops. We have 52

$$
\delta m_{\Sigma}^{2}=\frac{3 g_{S}^{4}}{\left(16 \pi^{2}\right)^{2}} m_{\Phi}^{2} \log \left(\frac{\Lambda^{2}}{m_{\Phi}^{2}}\right)
$$

with $\Lambda$ the mediation scale. We illustrate the size of the resulting tuning within the context of the simplified model (i.e. treating SM quartics as negligible) in Fig. 7. We set $\Lambda=50 \mathrm{TeV}$ and choose $m_{\Phi}^{2}=g_{S}^{2} u^{2} / 4$ so as to consider only parameters where the auxiliary Higgs quartic is unsuppressed. We also enforce the precision electroweak constraints as described above. This puts a lower bound on $u$ and therefore drives the fine-tuning. We see that the tuning becomes large in the decoupling limit, as well as the limit of large $g_{S}$, where the 2-loop effect Eq. (3.30) is enhanced. The tuning is less than $10 \%$ over most of the allowed parameter space. This is a significant 


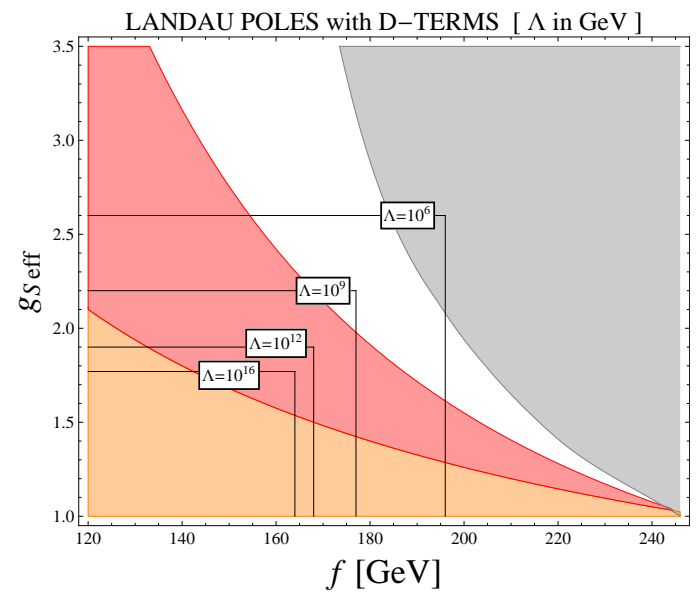

Fig. 8. Landau poles in the parameter space of the $D$-term model. The unshaded area is the allowed region, as in Fig. 3 .

difference between this mechanism and other proposed solutions to the Higgs tuning problem, which have locally small tuning only in a very specific range of parameters. In this model, the only requirement is that the $S U(2)_{S}$ breaking scale be close to the smallest phenomenologically allowed value. There are no other large mass hierarchies required in this model, and we conclude that there is no significant tuning in this model subject to only very mild restrictions of the parameters.

\subsection{Unification and Landau Poles}

The fact that the strong and electroweak guage couplings unify at high scales in the MSSM is a possible hint for the existence of SUSY in nature, and it is important to know to what extent unification is naturally incorporated into extensions of the MSSM such as the one we are considering. The extra fields in our model come in complete $S U(5)$ multiplets, but this by itself is not sufficient to ensure unification.

One issue is that the VEVs of the auxiliary Higgs fields break both $S U(2)_{S}$ and $S U(2)_{W}$, and therefore mix the gauge bosons from these groups and change the value of the measured weak gauge coupling. However, the correction is suppressed because of the large $S U(2)_{S}$ breaking from the VEVs of the fields $\Phi$ and $\bar{\Phi}$. The correction to the measured low-energy gauge coupling is of order $f^{4} / u^{4}$, which is negligibly small once we impose the $T$ paramter constraint.

Another issue is that this model requires that the couplings $g_{S}$ and $\lambda_{\Phi}$ be order unity at the TeV scale. Neither coupling is asymptotically free, so there is a danger 
that the running couplings become large below the GUT scale, potentially ruining gauge coupling unification. Also, large values of $f$ require a larger value of $y_{t}$ at the weak scale, so there is the danger of a Landau pole for this coupling as well. The maximum scale of perturbativity as a function of the parameters is indicated in Fig. 8 . As we expect, we find that a Landau pole appears at lower scales when either $g_{S}$ or $f$ gets large.

There is a Landau pole well below the GUT scale for all allowed parameters, and therefore this theory requires UV completion below that scale. Such UV completions can naturally preserve unification if the new physics comes in complete $S U(5)$ multiplets. An important point in the present model is that all fields with the large $g_{S}$ coupling satisfy exactly this GUT criterion. This makes it simple to have new physics that avoids the Landau pole in $g_{S}$ while preserving gauge coupling unification. As a simple illustrative example of this point, we consider a model where $S U(2)_{S}$ is embedded into a larger gauge group $S U(3)_{S^{\prime}}$ at a higher scale $M$. The particle content is given in Table 2. In order to break $S U(3)_{S^{\prime}} \rightarrow S U(2)_{S}$ we need the additional superpotential terms

$$
\Delta W \sim \lambda^{\prime} S^{\prime}\left(\bar{\Phi}^{\prime} \Phi^{\prime}-M^{2}\right)+F\left(\bar{\Delta} \Phi^{\prime}\right)+\bar{F}\left(\Delta \bar{\Phi}^{\prime}\right)
$$

where $S^{\prime}$ is a singlet. We assume that the scale $M$ is above the TeV scale, so the theory is approximately supersymmetric at the scale $M$. The $F$ - and $D$-flat conditions fix the VEVs

$$
\left\langle S^{\prime}\right\rangle=0, \quad\left\langle\Phi^{\prime}\right\rangle=\left\langle\bar{\Phi}^{\prime}\right\rangle=\left(\begin{array}{c}
0 \\
0 \\
M
\end{array}\right)
$$

The second and third terms in Eq. (3.31) generate masses of the form $\bar{F} \Delta_{3}$ and $F \bar{\Delta}_{3}$, with the additional components of the $\Delta$ fields matching onto $\Sigma_{u, d}$. The theory has an accidental $U(1)$ global symmetry under which $\Phi^{\prime}$ and $\bar{\Phi}^{\prime}$ have opposite charges, and there is therefore a massless singlet Goldstone chiral multiplet. All other fields get masses of order $M$, and the low energy theory is the model discussed above. The additional Goldstone multiplet can get masses from higher dimension operators, and is harmless.

Above the scale $M$, the $S U(3)_{S^{\prime}}$ gauge group has 7 flavors, and is therefore asymptotically free. In fact it is in the conformal window 53 55], and we expect that there is an IR-stable fixed point for the gauge coupling and Yukawa coupling $\lambda_{\Phi}$. It is therefore simple to find an RG trajectory where $g_{S}$ and $\lambda_{\Phi}$ are perturbative at the GUT scale, and run toward the fixed point in the IR. One possibility is that the scale $M$ is near but above the scale $\Lambda_{S^{\prime}}$ where the $S U(3)_{S^{\prime}}$ coupling becomes strong. This 


\begin{tabular}{c|cc} 
& $S U(3)_{S^{\prime}}$ & $S U(5)_{\mathrm{SM}}$ \\
\hline$\Phi, \Phi^{\prime}$ & $\square$ & 1 \\
$\bar{\Phi}, \bar{\Phi}^{\prime}$ & $\square$ & 1 \\
$\Delta$ & $\square$ & $\square$ \\
$\bar{\Delta}$ & $\square$ & $\square$ \\
$F$ & 1 & $\square$ \\
$\bar{F}$ & 1 & $\square$ \\
\hline
\end{tabular}

Table 2. Field content of the extended $D$-term model. The fields are in complete multiplets of $S U(5)_{\mathrm{SM}} \supset S U(3)_{C} \times S U(2)_{W} \times U(1)_{Y}$.

requires a coincidence of scales. We can avoid this coincidence if the $S U(3)_{S^{\prime}}$ coupling becomes strong and flows to its fixed point above the scale $M$. The operator $\bar{\Phi}^{\prime} \Phi^{\prime}$ has dimension $\frac{12}{7}$, and the coupling $\lambda^{\prime}$ in Eq. (3.31) therefore has a large anomalous dimension and becomes strong at a scale

$$
\Lambda_{\lambda^{\prime}} \sim \Lambda_{S^{\prime}}\left(\frac{\lambda^{\prime}\left(\Lambda_{S^{\prime}}\right)}{4 \pi}\right)^{7 / 2} .
$$

For $\lambda^{\prime}\left(\Lambda_{S^{\prime}}\right) \sim 0.1$ we obtain $\Lambda_{\lambda^{\prime}} / \Lambda_{S^{\prime}} \sim 10^{-7}$, so the coupling $\lambda^{\prime}$ can naturally remain perturbative at scales far below the scale where the $S U(3)_{S^{\prime}}$ fixed point is reached; the $F\left(\bar{\Delta} \Phi^{\prime}\right)$ and $\bar{F}\left(\Delta \bar{\Phi}^{\prime}\right)$ couplings behave in the same way. We therefore consider the case where the superpotential couplings break the $S U(3)_{S^{\prime}}$ at scales below $\Lambda_{S^{\prime}}$ but above the scale where $\lambda^{\prime}$ gets strong. In this regime, the $S^{\prime}$ term in the superpotential does not have a large anomalous dimension, and the $S U(3)_{S^{\prime}}$ breaking scale is given by

$$
\tilde{M} \sim M^{7 / 6} \Lambda_{S^{\prime}}^{-1 / 6}
$$

This is very insensitive to the scale $\Lambda_{S^{\prime}}$, so the breaking scale is still essentially set by $M$. The bottom line is that there is a large regime of parameters where the RG trajectory reaches the strong $S U(3)_{S^{\prime}}$ fixed point, and the theory breaks to the $S U(2)_{S}$ theory at a scale set by the parameter $M$. This theory is strongly coupled, so we can naturally obtain a large value of the $S U(2)_{S}$ gauge coupling at the weak scale. The salient features of this scenario are sketched in Fig. 9 .

Above the scale $M$, the gauge group has 4 additional $\mathbf{5} \oplus \overline{\mathbf{5}}$ compared to the MSSM, and the SM gauge couplings remain perturbative up to the GUT scale. In the range of scales where the $S U(3)_{S^{\prime}}$ gauge coupling is at a strong fixed point, the beta functions of the SM gauge couplings are reduced compared to the tree-level ones, making the couplings even more perturbative: each of the 3 strongly coupled flavors 

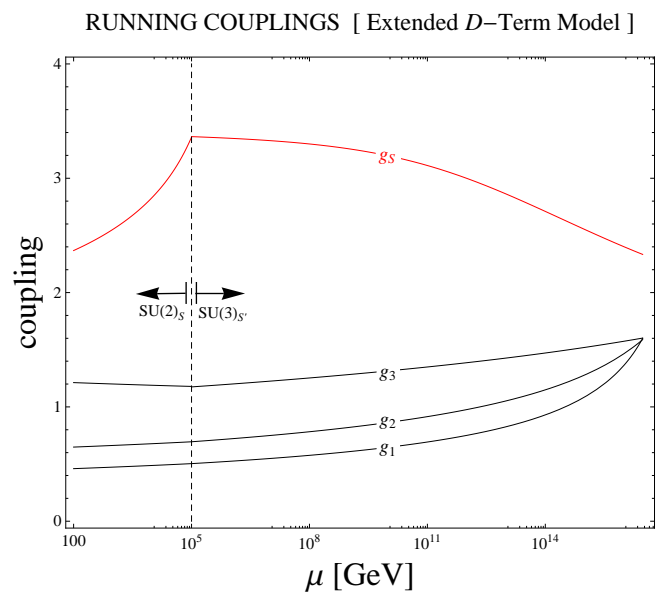

Fig. 9. Running couplings and their matching at the scale $M \sim 10^{5} \mathrm{GeV}$ where $S U(3)_{S^{\prime}}$ is broken to $S U(2)_{S}$. We take $f=160 \mathrm{GeV}$ such that the top coupling remains perturbative and the tuning required is $\mathcal{O}(15 \%)$.

counts as $\frac{5}{7}$ flavors in the RG equations for the SM gauge couplings.

We must take $f \lesssim 160 \mathrm{GeV}$ in order to avoid a Landau pole for $y_{t}$, which requires $g_{S} \gtrsim 2.5$ at the weak scale (see Fig. 3). This is perfectly natural in this model if the $S U(3)_{S^{\prime}}$ coupling is at its fixed point down to scales of order $100 \mathrm{TeV}$. We note that this puts the model in an interesting parameter regime that favors an induced tadpole, and therefore a small value of the Higgs cubic coupling.

This model is only an example. We can imagine other kinds of new physics that replaces the Landau pole of this coupling, such as compositeness or extra dimensions. Such a UV completion preserves unification as long as all new fields are in complete $S U(5)$ multiplets and there are not too many of them. It is also possible to have larger values of $f$ if the top quark is composite, as in the models of Ref. [56]. These models generate complete composite GUT multiplets, and therefore also preserve unification.

\subsection{Discussion}

The essential ingredient in this model is the presence of additional 'auxiliary' Higgs fields charged under a new non-abelian gauge group $S U(2)_{S}$. The new gauge coupling can easily be larger than the electroweak gauge couplings at the $\mathrm{TeV}$ scale, so these Higgs fields naturally have a large quartic coupling. This model can be viewed as a perturbative (and hence calculable) version of the mechanism of 'superconformal technicolor' proposed in Ref. [32, 33]. In those models, the auxiliary Higgs fields 
are composites arising from strong confining dynamics at the $\mathrm{TeV}$ scale, similar to technicolor. The presence of strong dynamics at the same scale as the SUSY breaking scale is not a coincidence if the strong dynamics is conformal above the TeV scale, and confinement and electroweak symmetry breaking are triggered by soft SUSY breaking. Precision electroweak corrections are not precisely calculable in this model, but using 'naïve dimensional analysis' estimates place them near the edge of the $95 \%$ confidence level constraints.

The present perturbative models are fully calculable, so we can check all experimental constraints without large theoretical uncertainties. But it is not at all clear that nature cares about whether we can calculate. One sense in which the present models are an improvement over the strongly-coupled models is that they are compatible with perturbative unification. This comes at a price however, since putting the auxiliary Higgs fields in a $\mathbf{5} \oplus \overline{\mathbf{5}}$ of $S U(5)$ means that their VEVs break custodial symmetry. $t^{2}$ This necessitates a little hierarchy between the $S U(2)_{S}$ breaking scale $(\sim \mathrm{TeV})$ and the Higgs mass scale $(\sim 100 \mathrm{GeV})$. If we did not require gauge coupling unification, we could make a simpler model where the auxiliary Higgs fields are $S U(2)_{W}$ triplets, but we will not pursue this here.

In our discussion, we have been agnostic about the superpartner spectrum. In order to have a natural model, this spectrum must have a relatively light stop and gluino, and this is compatible with experimental limits only for special spectra, such as a somewhat compressed spectrum. We do not address how this arises, since the phenomenological issues essentially factorize: our model is compatible with any 'natural' spectrum that is not ruled out by the data.

\section{F-term Models}

Next we consider the possibility that the quartic coupling of $\Sigma$ is generated by $F$ terms via an NMSSM-like superpotential interaction

$$
\Delta W=\lambda_{S} S \Sigma_{u} \Sigma_{d}
$$

where $S$ is a singlet and $\Sigma_{u, d}$ are Higgs doublets. If we require perturbativity up to a high scale such as the GUT scale, the coupling $\lambda_{S}$ can be somewhat larger than the corresponding coupling in the NMSSM because the top Yukawa coupling does not contribute to the leading order RG running of $\lambda_{S}$. The largest value of the singlet

${ }^{2}$ The order parameter used in the superconformal technicolor models in Ref. 32,33 was a $(2,2)$ of $S U(2)_{L} \times S U(2)_{R}$, which preserves custodial symmetry but does not have a simple embedding into a complete GUT multiplet. 
coupling compatible with perturbativity up to the GUT scale is approximately $\lambda_{S}=$ 0.92 . We will see that this is not large enough to make a realistic model of induced EWSB, so we will also discuss a hybrid model with both $D$ and $F$-term contributions to the auxiliary Higgs quartic that satisfies all phenomenological constraints and is perturbative up to the GUT scale.

The $F$-term model is interesting also because it is has different phenomenology than the $D$-term models. One important difference is that it requires a nonvanishing VEV for both $\Sigma_{u}$ and $\Sigma_{d}$ in order for the quartic Eq. 4.1) to play a role in EWSB. We consider the case where the VEVs are given by

$$
\left\langle\Sigma_{u}\right\rangle=\frac{1}{\sqrt{2}}\left(\begin{array}{cc}
0 & 0 \\
f_{u} & 0
\end{array}\right) ; \quad\left\langle\Sigma_{d}\right\rangle=\frac{1}{\sqrt{2}}\left(\begin{array}{cc}
0 & f_{d} \\
0 & 0
\end{array}\right) .
$$

We will make the simplifying assumption that $\langle S\rangle=0$. Imposing this exactly is unnatural, since gaugino loops will generate a nonzero $A$ term of the form $S \Sigma_{u} \Sigma_{d}$, and this will give a tadpole for $S$. However, $\langle S\rangle$ can be suppressed if the $S$ mass-squared is somewhat larger than the other soft masses, so this can be a good approximation. This can be motivated phenomenologically from the fact that a large VEV for $S$ correlates with large mixing between $H$ and $S$, which is constrained by the measured Higgs couplings. We stress however that we are making this assumption mainly for simplicity. We believe that allowing $\langle S\rangle \neq 0$ will not significantly change the main conclusions below.

With this assumption, the potentially relevant terms in the superpotential are

$$
\Delta W=\mu H_{u} H_{d}+\lambda_{u} H_{u} \Sigma_{d} \Phi+\lambda_{d} H_{d} \Sigma_{u} \Phi+\bar{\lambda}_{u} H_{u} \Sigma_{d} \bar{\Phi}+\bar{\lambda}_{d} H_{d} \Sigma_{u} \bar{\Phi} .
$$

as well as soft SUSY breaking terms

$$
\begin{aligned}
\Delta V= & m_{H_{u}}^{2}\left|H_{u}\right|^{2}+m_{H_{d}}^{2}\left|H_{d}\right|^{2}+m_{\Sigma_{u}}\left|\Sigma_{u}\right|^{2}+m_{\Sigma_{d}}\left|\Sigma_{d}\right|^{2} \\
& +B \mu H_{u} H_{d}+B_{S} \Sigma_{u} \Sigma_{d}+B_{u} H_{u} \Sigma_{d}+B_{d} H_{d} \Sigma_{u}+\text { h.c. } \\
& +A \text { terms. }
\end{aligned}
$$

The fact that we need an explicit $\mu$ term is due to our simplifying assumption that $\langle S\rangle=0$. We further neglect the couplings $\lambda_{u, d}$ and $\bar{\lambda}_{u, d}$ and the corresponding $A$ terms, also for simplicity. For completeness we quote the resulting minimization 
conditions:

$$
\begin{gathered}
m_{H_{d}}^{2}=B \mu \tan \beta-B_{d} \frac{s_{\beta_{\Sigma}}}{c_{\beta}} \cot \gamma-\frac{1}{2} m_{Z}^{2}\left(s_{\gamma}^{2} \cdot \cos 2 \beta+c_{\gamma}^{2} \cdot \cos 2 \beta_{\Sigma}\right) \\
m_{H_{u}}^{2}=B \mu \cot \beta+B_{u} \frac{c_{\beta_{\Sigma}}}{s_{\beta}} \cot \gamma+\frac{1}{2} m_{Z}^{2}\left(s_{\gamma}^{2} \cdot \cos 2 \beta+c_{\gamma}^{2} \cdot \cos 2 \beta_{\Sigma}\right) \\
m_{\Sigma_{d}}^{2}=B_{s} \tan \beta_{\Sigma}+B_{u} \frac{s_{\beta}}{c_{\beta_{\Sigma}}} \tan \gamma-\frac{1}{2} m_{Z}^{2}\left(s_{\gamma}^{2} \cdot \cos 2 \beta+c_{\gamma}^{2} \cdot \cos 2 \beta_{\Sigma}\right) \\
\quad-\frac{1}{2} \lambda_{S}^{2} v^{2} c_{\beta_{\Sigma}}^{2} c_{\gamma}^{2} \\
m_{\Sigma_{u}}^{2}=B_{s} \cot \beta_{\Sigma}-B_{d} \frac{c_{\beta}}{s_{\beta_{\Sigma}}} \tan \gamma+\frac{1}{2} m_{Z}^{2}\left(s_{\gamma}^{2} \cdot \cos 2 \beta+c_{\gamma}^{2} \cdot \cos 2 \beta_{\Sigma}\right) \\
-\frac{1}{2} \lambda_{S}^{2} v^{2} c_{\beta_{\Sigma}}^{2} c_{\gamma}^{2}
\end{gathered}
$$

where

$$
\tan \beta_{\Sigma}=\frac{f_{u}}{f_{d}}
$$

As in $\S 3$ we consider a simplified case where $v_{d} \ll v_{u}, \sqrt{f_{u}^{2}+f_{d}^{2}}$, (i.e. large $\tan \beta$ ). We have a mass matrix in the basis $\left(H_{u}^{0}, \Sigma_{u}^{0}, \tilde{\Sigma}_{d}^{0}\right)$ given by (denoting $\lambda_{S}=\lambda$ )

$$
\mathcal{M}^{2}=\left(\begin{array}{ccc}
m_{H_{u}}^{2} & 0 & -\frac{m_{H_{u}}^{2} v_{u}}{f_{d}} \\
0 & \frac{1}{2} \lambda^{2} f_{d}^{2}+\frac{m_{\Sigma_{d}}^{2} f_{d}^{2}}{f_{u}^{2}}-\frac{m_{H_{u}}^{2} v_{u}^{2}}{f_{u}^{2}} & \frac{1}{2} \lambda^{2} f_{u} f_{d}-\frac{m_{\Sigma_{d}}^{2} f_{d}}{f_{u}}+\frac{m_{H_{u}}^{2} v_{u}^{2}}{f_{u} f_{d}} \\
-\frac{m_{H_{u}}^{2} v_{u}}{f_{d}} & \frac{1}{2} \lambda^{2} f_{u} f_{d}-\frac{m_{\Sigma_{d}}^{2} f_{d}}{f_{u}}+\frac{m_{H_{u}}^{2} v_{u}^{2}}{f_{u} f_{d}} & \frac{1}{2} \lambda^{2} f_{u}^{2}+m_{\Sigma_{d}}^{2}
\end{array}\right) .
$$

This simplified model has 9 parameters, given by the mass terms in Eq. (4.4) and the superpotential coupling $\lambda_{S}$. We trade 5 of these parameters for the VEVs $v_{u, d}$ and $f_{u, d}$ and the lightest Higgs mass and scan over the remaining parameters. We display the results in the plane of $\lambda_{S}$ and $f=\sqrt{f_{u}^{2}+f_{d}^{2}}$ in Fig. 10. As we expect, the results are qualitatively similar to both the simplified model and the $D$-term model. In particular, the Higgs VEV is dominated by an induced tadpole for roughly half of the allowed parameter space. The Landau poles generally occur at lower scales in this model than in the $D$-term model. One important difference is that $\lambda_{S}$ coupling runs at one loop, while the gauge coupling $g_{S}$ in the $D$-term model runs only at 2 loops. For $y_{t}$ there are two effects. First, $y_{t}$ must typically be larger in the $F$-term models because both $f_{u, d}$ are nonzero. Second, there are 2 additional $\mathbf{5} \oplus \overline{\mathbf{5}}$ in the $D$-term model, and only one in the $F$-term model. This makes the $S U(3)_{C}$ gauge coupling larger at high scales in the $D$-term model, slowing the running of $y_{t}$. We could of course add additional $\mathbf{5} \oplus \overline{\mathbf{5}}$ to the $F$-term model, but we will not explore this here.

To get a model compatible with perturbative unification, we turn to a 'hybrid' model where both $F$ and $D$ terms contribute to the auxiliary Higgs quartic. In the 

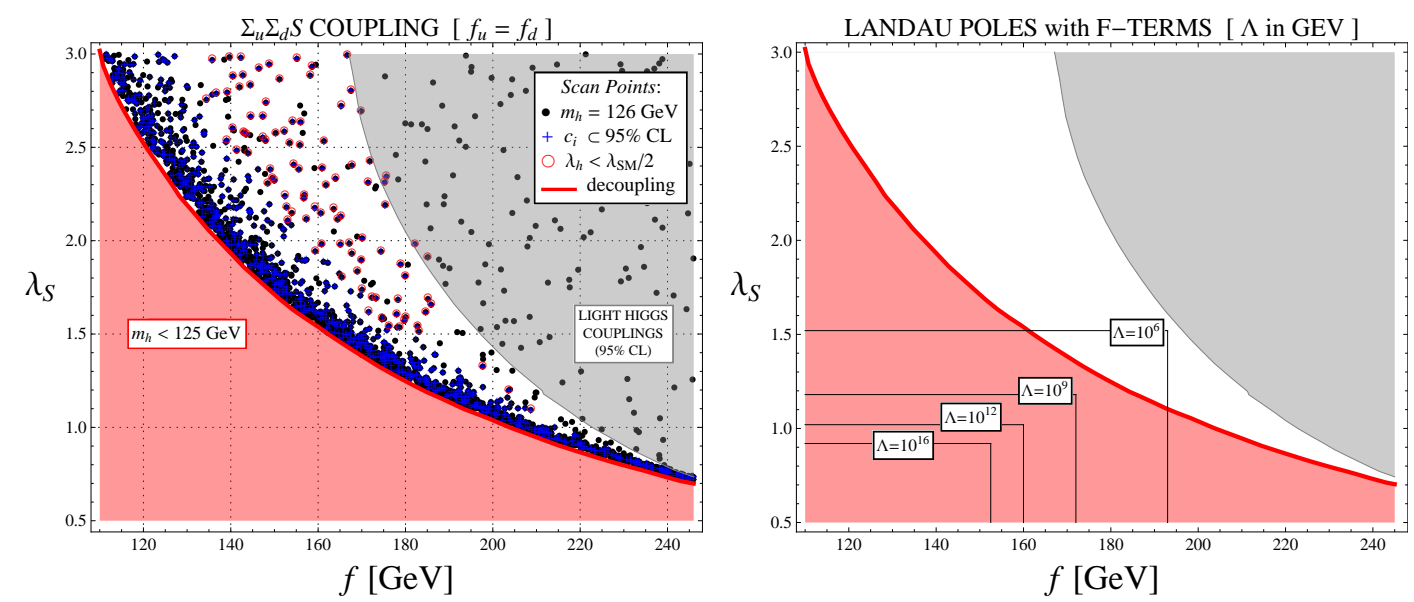

Fig. 10. Parameter space of the model with non-decoupling F-terms. Left: Numerical scan of parameter space; the points are labeled as in Fig. 4. Right: Position of Landau poles (compare Fig. 8).

model we consider, we choose an intermediate ratio of auxiliary $\mathrm{VEVs}, \tan \beta_{\Sigma}=2.5$, which allows the singlet coupling $\lambda_{S}$ to contribute significantly to the auxiliary quartic. The weak scale value of $\lambda_{S}$ can be larger than in the pure $F$-term model because $g_{S}$ gives a negative contribution to the $\lambda_{S}$ beta function. The resulting picture is shown in Fig. 11. We see that we get a model that is perturbative up to the GUT scale only if the model is close to the decoupling limit. This may be regarded as a kind of tuning, but see the discussion below. Models with additional structure below the GUT scale may be a more attractive possibility to combine naturalness with unification. As in the $D$-term models, the auxiliary Higgs fields naturally come in complete $S U(5)$ multiplets, so UV completing these models in a manner that preserves gauge coupling unification should be possible, but we will not attempt to address that here.

We conclude by commenting on the naturalness of the $F$-term models discussed above. Models with $D$-terms have a tree-level violation of custodial symmetry from the $\Sigma$ VEVs. This is absent in the pure $F$-term model, and so this model requires no little hierarchy among the masses, and therefore there is no danger of fine-tuning. This is certainly a very attractive feature of this model. In the hybrid model, we must have a little hierarchy to avoid large $T$-parameter corrections, but the value of $g_{S}$ is smaller, so the tuning is reduced compared to the pure $D$-term model. 


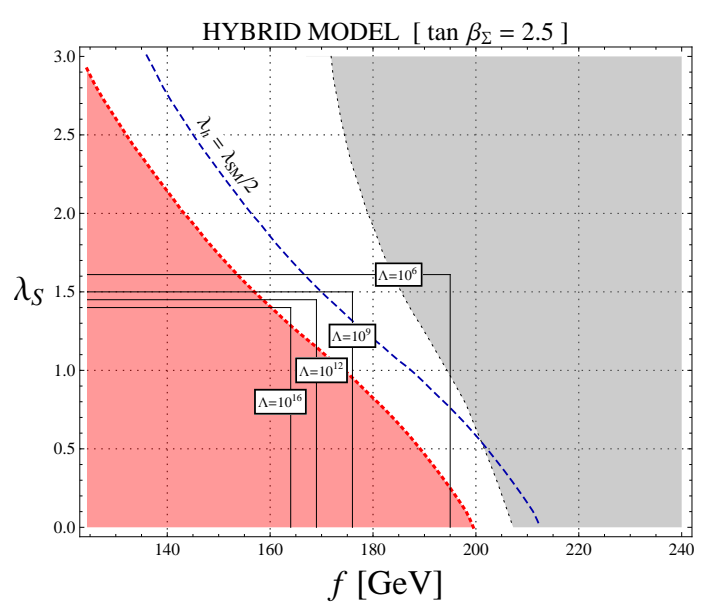

Fig. 11. Allowed region and Landau poles (corresponding to a given point's cutoff) for a hybrid model with $F$ and $D$ terms. The unshaded area corresponds to the allowed space, and cutoff values are quoted in GeV. The position of the Landau poles is determined by optimizing the value of the couplings $g_{S}\left(\mu=m_{W}\right)$ and $\lambda_{\Phi}\left(\mu=m_{W}\right)$ at each point.

\section{Conclusions}

We have presented SUSY models that address the Higgs naturalness problem. In these models the dominant source of electroweak symmetry breaking is due to the VEVs of the MSSM Higgs fields $H_{u, d}$, but there is a subleading contribution to electroweak symmetry breaking from additional 'auxiliary' Higgs fields. That is, we have

$$
m_{W}^{2}=\frac{1}{4} g^{2}\left(v_{u}^{2}+v_{d}^{2}+f^{2}\right),
$$

where $f$ arises from the auxiliary Higgs sector. A typical value is $f=150 \mathrm{GeV}$, which gives $\sqrt{v_{u}^{2}+v_{d}^{2}}=195 \mathrm{GeV}$. The auxiliary Higgs fields have no Yukawa couplings, which allows them to have a large quartic coupling either from additional gauge interactions or superpotential interactions. The masses of the auxiliary Higgs bosons are therefore above $125 \mathrm{GeV}$, even though their contribution to electroweak symmetry breaking is subleading. One simple limit of this model is the decoupling limit, where the heavy mass eigenstate has a very small VEV. In this limit, integrating out the auxiliary Higgs fields generates an induced quartic for the MSSM Higgs fields that can explain the observed $125 \mathrm{GeV}$ Higgs particle. The light Higgs has standard modellike couplings in the decoupling limit, so this limit is compatible with all the current Higgs data.

However, the data allows significant deviations from the decoupling limit. In fact, we can access another limit where the heavy auxiliary Higgs mass eigenstate has a 
significant VEV. In this case, electroweak symmetry is nonlinearly realized in the effective theory below the auxiliary Higgs mass. This allows the effective theory to contain relevant electroweak breaking terms such as tadpole terms for the light Higgs fields. If this tadpole is sufficiently large, the light Higgs VEVs and masses are very insensitive to the quartic coupling of the light Higgs. This means that the quartic (and cubic) coupling of the Higgs can be much smaller than those of the standard model Higgs. This is a smoking-gun signal of this mechanism.

Between these two limits there is a large and phenomenologically interesting parameter space. Importantly, the tuning is less than $10 \%$ in almost all of the allowed parameter space, making this solution to the tuning problem more robust than many others considered in the literature.

These models have rich phenomenology at the LHC and beyond. First, the Higgs couplings can have significant deviation from their standard-model values away from the decoupling limit. Away from this limit, the auxiliary Higgs particles can be light, and mix significantly with the light Higgs fields. The auxiliary Higgs fields have suppressed couplings to fermions and gauge bosons, but can have appreciable production cross sections. They can decay to electroweak gauge bosons and/or the $125 \mathrm{GeV}$ Higgs. Current standard Higgs searches in the heavy mass region however do not yet have sufficient sensitivity to constrain the multi-Higgs models presented here: considering a benchmark mass of $350 \mathrm{GeV}$ for the heavy state and assuming all final states are the same as those of the light Higgs, the current exclusions reach no lower than $\mu=\Gamma / \Gamma_{\mathrm{SM}} \simeq 0.2$ combining all $H \rightarrow V V$ channels, corresponding to $H$ couplings of order $45 \%$ or less of the corresponding couplings of the light Higgs [57]. This is easily compatible with light Higgs couplings that are within $10 \%$ of the SM, so both direct and indirect probes of the heavy states still allow for their (undetected) existence. Moreover, there can be a significant branching fraction for heavy Higgs to decay to lighter Higgs states, such as $H \rightarrow h h$ if the heavy states are not decoupled [58], which further weaken direct search bounds. The detailed bounds depend on the full parameter space of the model, not just the 2-parameter subspace emphasized here, and goes beyond the scope of this study.

Another important point for Higgs phenomenology is that the light Higgs quartic (and hence cubic) coupling can easily be highly suppressed compared to the standard model. Because of the destructive interference with direct double Higgs production, this gives an increased rate for double Higgs production compared to the standard model, which may be observable at the $14 \mathrm{TeV}$ LHC with $300 \mathrm{fb}^{-1}[45,47]$ and may provide additional evidence for the class of models studied here.

Finally, there is the rest of the superpartner spectrum. The present model is 
motivated by naturalness, so the stops cannot be too heavy in this model. Minimal predictive models of SUSY breaking (such as gauge mediation, gaugino mediation, or anomaly mediation) do not give a natural allowed SUSY spectrum, so we simply treat the soft SUSY breaking terms as phenomenological parameters. The superpartner spectrum is constrained by the absence of a signal in SUSY searches so far. There are a number of ways that this could happen, including (but not limited to) $m_{\tilde{t}} \sim m_{t}$, a compressed spectrum, $R$-parity violation, or decays through a hidden sector [59]. Searches at the $14 \mathrm{TeV}$ LHC will have a large reach in these scenarios, and we fervently hope for a signal there.

\section{Acknowledgements}

We have benefited from discussions with A. Azatov, R. Contino, N. Craig, C. Englert, J. Terning, and N. Weiner. M.L. and Y.T. are supported by the Department of Energy under grant DE-FG02-91ER40674. J.G. is supported by the ERC Advanced Grant No. 267985. Y.Z. is supported by SLAC, which is operated by Stanford University for the US Department of Energy under contract DE-AC02-76SF00515.

\section{Appendix: Renormalization Group for Auxiliary Higgs Models}

Various order-1 couplings are required in each of the models presented. In practice we run couplings, including SM gauge couplings, at two loops with results that have been verified using the package SARAH 60,61]. However, we summarize here the leading RG equations for three separate cases to facilitate simple checks of the results: $i$ ) auxiliary quartics arising from $D$ terms alone; $i$ ) quartics from $F$ terms alone; and iii) quartics from both $F$ and $D$ terms (i.e. the 'hybrid' model). We have the following:

$$
\begin{aligned}
& D \text {-term model: }\left\{\begin{array}{l}
b_{g_{S}}^{(2)}=2 g_{S}^{3}\left(g_{1}^{2}+3 g_{2}^{2}+8 g_{3}^{2}+9 g_{S}^{2}-\lambda_{\Phi}^{2}\right), \\
b_{\lambda_{\Phi}}^{(1)}=\lambda_{\Phi}\left(4 \lambda_{\Phi}^{2}-3 g_{S}^{2}\right),
\end{array}\right. \\
& \text { F-term model: } \quad\left\{b_{\lambda_{S}}^{(1)}=\lambda_{S}\left(4 \lambda_{S}^{2}-\frac{3}{5} g_{1}^{2}-3 g_{2}^{2}\right)\right. \text {, } \\
& \text { Hybrid model: }\left\{\begin{array}{l}
b_{g_{S}}^{(2)}=2 g_{S}^{3}\left(g_{1}^{2}+3 g_{2}^{2}+8 g_{3}^{2}+9 g_{S}^{2}-\lambda_{\Phi}^{2}-2 \lambda_{S}^{2}\right), \\
b_{\lambda_{\Phi}}^{(1)}=\lambda_{\Phi}\left(4 \lambda_{\Phi}^{2}-3 g_{S}^{2}\right), \\
b_{\lambda_{S}}^{(1)}=\lambda_{S}\left(6 \lambda_{S}^{2}-\frac{3}{5} g_{1}^{2}-3 g_{2}^{2}-3 g_{S}^{2}\right),
\end{array}\right.
\end{aligned}
$$

with $\beta_{g} \equiv d g / d \ln \mu=\sum_{\ell} b_{g}^{(\ell)} /\left(16 \pi^{2}\right)^{\ell}$. In the case of the extended $D$ term model where $S U(2)_{S}$ is embedded into the larger $S U(3)_{S^{\prime}}$ at an intermediate scale $M$, we 
have leading RG running in the (weakly-coupled) UV governed by

$$
\begin{aligned}
b_{\lambda_{\Phi_{1,2}}}^{(1)} & =\lambda_{\Phi_{1,2}}\left(5 \lambda_{\Phi_{1,2}}^{2}+3 \lambda_{\Phi_{2,1}}^{2}-\frac{16}{3} g_{S^{\prime}}^{2}\right), \\
b_{g_{S}}^{(1)} & =-2 g_{S}^{3}, \\
b_{g_{S}}^{(2)} & =g_{S^{\prime}}^{3}\left(2 g_{1}^{2}+6 g_{2}^{2}+16 g_{3}^{2}+\frac{76}{3} g_{S^{\prime}}^{2}-2\left(\lambda_{\Phi_{1}}^{2}+\lambda_{\Phi_{2}}^{2}\right)\right) .
\end{aligned}
$$

Here the superpotential couplings are given by $W=\lambda_{\Phi_{1}} S \bar{\Phi}^{\prime} \Phi^{\prime}+\lambda_{\Phi_{2}} S \bar{\Phi} \Phi$. When using these equations, it is important to take into account the fact that there are extra multiplets on the running of the standard model gauge couplings, which also affects the running of the top quark Yukawa coupling.

\section{References}

[1] S. Chatrchyan et al. [CMS Collaboration], "Observation of a new boson at a mass of 125 gev with the CMS experiment at the LHC," Phys.Lett. B716 (2012) 30-61, arXiv:1207.7235 [hep-ex].

[2] G. Aad et al. [ATLAS Collaboration], "Observation of a new particle in the search for the standard model higgs boson with the ATLAS detector at the LHC," Phys.Lett. B716 1-29, arXiv: 1207.7214 [hep-ex].

[3] "Combined coupling measurements of the higgs-like boson with the ATLAS detector using up to $25 \mathrm{fb}^{-1}$ of proton-proton collision data," Tech. Rep. ATLASCONF-2013-034, CERN, Geneva, Mar, 2013.

[4] "Combination of standard model higgs boson searches and measurements of the properties of the new boson with a mass near $125 \mathrm{GeV}$," Tech. Rep. CMS-PASHIG-12-045, CERN, Geneva, 2012.

[5] J. R. Ellis, G. Ridolfi, and F. Zwirner, "On radiative corrections to supersymmetric Higgs boson masses and their implications for LEP searches," Phys.Lett. B262 (1991) 477-484.

[6] H. E. Haber and R. Hempfling, "Can the mass of the lightest Higgs boson of the minimal supersymmetric model be larger than $\mathrm{m}(\mathrm{Z})$ ?," Phys.Rev.Lett. 66 (1991) 1815-1818.

[7] Y. Okada, M. Yamaguchi, and T. Yanagida, "Upper bound of the lightest Higgs boson mass in the minimal supersymmetric standard model," Prog.Theor.Phys. 85 (1991) 1-6. 
[8] A. Brignole, "Radiative corrections to the supersymmetric neutral Higgs boson masses," Phys.Lett. B281 (1992) 284-294.

[9] M. S. Carena, J. Espinosa, M. Quiros, and C. Wagner, "Analytical expressions for radiatively corrected Higgs masses and couplings in the MSSM," Phys.Lett. B355 (1995) 209-221, arXiv:hep-ph/9504316 [hep-ph].

[10] A. Birkedal, Z. Chacko, and Y. Nomura, "Relaxing the upper bound on the mass of the lightest supersymmetric Higgs boson," Phys.Rev. D71 (2005) 015006. arXiv:hep-ph/0408329 [hep-ph].

[11] H. P. Nilles, M. Srednicki, and D. Wyler, "Weak Interaction Breakdown Induced by Supergravity," Phys.Lett. B120 (1983) 346.

[12] J. R. Ellis, J. Gunion, H. E. Haber, L. Roszkowski, and F. Zwirner, "Higgs Bosons in a Nonminimal Supersymmetric Model," Phys.Rev. D39 (1989) 844.

[13] J. Espinosa and M. Quiros, "Upper bounds on the lightest Higgs boson mass in general supersymmetric Standard Models," Phys.Lett. B302 (1993) 51-58, arXiv:hep-ph/9212305 [hep-ph]

[14] G. L. Kane, C. F. Kolda, and J. D. Wells, "Calculable upper limit on the mass of the lightest Higgs boson in any perturbatively valid supersymmetric theory," Phys.Rev.Lett. 70 (1993) 2686-2689, arXiv:hep-ph/9210242 [hep-ph].

[15] J. Cao and J. M. Yang, "Current experimental constraints on NMSSM with large lambda," Phys.Rev. D78 (2008) 115001, arXiv:0810.0989 [hep-ph].

[16] L. Cavicchia, R. Franceschini, and V. S. Rychkov, "Supersymmetry without a light Higgs boson at the CERN LHC," Phys.Rev. D77 (2008) 055006, arXiv:0710.5750 [hep-ph].

[17] R. Barbieri, E. Bertuzzo, M. Farina, P. Lodone, and D. Pappadopulo, "A Non Standard Supersymmetric Spectrum," JHEP 1008 (2010) 024, arXiv:1004.2256 [hep-ph].

[18] P. Lodone, "Naturalness bounds in extensions of the MSSM without a light Higgs boson," JHEP 1005 (2010) 068, arXiv:1004.1271 [hep-ph].

[19] R. Franceschini and S. Gori, "Solving the $\mu$ problem with a heavy Higgs boson," JHEP 1105 (2011) 084, arXiv: 1005.1070 [hep-ph]. 
[20] J.-J. Cao, Z.-X. Heng, J. M. Yang, Y.-M. Zhang, and J.-Y. Zhu, "A SM-like Higgs near $125 \mathrm{GeV}$ in low energy SUSY: a comparative study for MSSM and NMSSM," JHEP 1203 (2012) 086, arXiv:1202.5821 [hep-ph].

[21] L. J. Hall, D. Pinner, and J. T. Ruderman, "A Natural SUSY Higgs Near 126 GeV," JHEP 1204 (2012) 131, arXiv:1112.2703 [hep-ph].

[22] P. Batra, A. Delgado, D. E. Kaplan, and T. M. Tait, "The Higgs mass bound in gauge extensions of the minimal supersymmetric standard model," JHEP 0402 (2004) 043, arXiv:hep-ph/0309149 [hep-ph].

[23] A. Maloney, A. Pierce, and J. G. Wacker, "D-terms, unification, and the Higgs mass," JHEP 0606 (2006) 034, arXiv:hep-ph/0409127 [hep-ph].

[24] R. Harnik, G. D. Kribs, D. T. Larson, and H. Murayama, "The Minimal supersymmetric fat Higgs model," Phys.Rev. D70 (2004) 015002, arXiv:hep-ph/0311349 [hep-ph].

[25] S. Chang, C. Kilic, and R. Mahbubani, "The New fat Higgs: Slimmer and more attractive," Phys.Rev. D71 (2005) 015003, arXiv:hep-ph/0405267 [hep-ph].

[26] J. D. Wells, "Implications of supersymmetry breaking with a little hierarchy between gauginos and scalars," arXiv:hep-ph/0306127 [hep-ph].

[27] G. Giudice and A. Romanino, "Split supersymmetry," Nucl.Phys. B699 (2004) 65-89, arXiv:hep-ph/0406088 [hep-ph].

[28] N. Arkani-Hamed and S. Dimopoulos, "Supersymmetric unification without low energy supersymmetry and signatures for fine-tuning at the LHC," JHEP 0506 (2005) 073, arXiv:hep-th/0405159 [hep-th].

[29] G. F. Giudice and A. Strumia, "Probing High-Scale and Split Supersymmetry with Higgs Mass Measurements," Nucl.Phys. B858 (2012) 63-83, arXiv:1108.6077 [hep-ph].

[30] N. Arkani-Hamed, A. Gupta, D. E. Kaplan, N. Weiner, and T. Zorawski, "Simply Unnatural Supersymmetry," arXiv:1212.6971 [hep-ph].

[31] A. Arvanitaki, N. Craig, S. Dimopoulos, and G. Villadoro, "Mini-Split," JHEP 1302 (2013) 126, arXiv:1210.0555 [hep-ph]. 
[32] A. Azatov, J. Galloway, and M. A. Luty, "Superconformal Technicolor: Models and Phenomenology," Phys.Rev. D85 (2012) 015018, arXiv:1106.4815 [hep-ph].

[33] A. Azatov, J. Galloway, and M. A. Luty, "Superconformal Technicolor," Phys.Rev.Lett. 108 (2012) 041802, arXiv:1106.3346 [hep-ph].

[34] N. Craig, D. Stolarski, and J. Thaler, "A Fat Higgs with a Magnetic Personality," JHEP 1111 (2011) 145, arXiv:1106.2164 [hep-ph].

[35] T. Gherghetta and A. Pomarol, "A Distorted MSSM Higgs Sector from LowScale Strong Dynamics," JHEP 1112 (2011) 069, arXiv:1107.4697 [hep-ph].

[36] J. J. Heckman, P. Kumar, C. Vafa, and B. Wecht, "Electroweak Symmetry Breaking in the DSSM," JHEP 1201 (2012) 156, arXiv:1108.3849 [hep-ph].

[37] N. Arkani-Hamed, A. G. Cohen, and H. Georgi, "Electroweak symmetry breaking from dimensional deconstruction," Phys.Lett. B513 (2001) 232-240, arXiv:hep-ph/0105239 [hep-ph].

[38] N. Arkani-Hamed, A. Cohen, E. Katz, and A. Nelson, "The Littlest Higgs," JHEP 0207 (2002) 034, arXiv:hep-ph/0206021 [hep-ph].

[39] R. Contino, Y. Nomura, and A. Pomarol, "Higgs as a holographic pseudoGoldstone boson," Nucl.Phys. B671 (2003) 148-174, arXiv:hep-ph/0306259 [hep-ph].

[40] S. Chang and J. G. Wacker, "Little Higgs and custodial SU(2)," Phys.Rev. D69 (2004) 035002, arXiv:hep-ph/0303001 [hep-ph].

[41] K. Agashe, R. Contino, and A. Pomarol, "The Minimal composite Higgs model," Nucl.Phys. B719 (2005) 165-187, arXiv:hep-ph/0412089 [hep-ph].

[42] D. S. Alves, P. J. Fox, and N. Weiner, "Supersymmetry with a Sister Higgs," arXiv:1207.5522 [hep-ph].

[43] R. Barbieri and G. Giudice, "Upper Bounds on Supersymmetric Particle Masses," Nucl.Phys. B306 (1988) 63.

[44] A. Azatov and J. Galloway, "Electroweak Symmetry Breaking and the Higgs Boson: Confronting Theories at Colliders," Int.J.Mod.Phys. A28 (2013) 1330004, arXiv:1212.1380 [hep-ph]. 
[45] M. Klute, R. Lafaye, T. Plehn, M. Rauch, and D. Zerwas, "Measuring Higgs Couplings from LHC Data," Phys.Rev.Lett. 109 (2012) 101801, arXiv:1205.2699 [hep-ph].

[46] M. J. Dolan, C. Englert, and M. Spannowsky, "Higgs self-coupling measurements at the LHC," JHEP 1210 (2012) 112, arXiv:1206.5001 [hep-ph].

[47] F. Goertz, A. Papaefstathiou, L. L. Yang, and J. Zurita, "Higgs Boson selfcoupling measurements using ratios of cross sections," JHEP 1306 (2013) 016, arXiv:1301.3492 [hep-ph].

[48] M. E. Peskin and T. Takeuchi, "A New constraint on a strongly interacting Higgs sector," Phys.Rev.Lett. 65 (1990) 964-967.

[49] M. E. Peskin and T. Takeuchi, "Estimation of oblique electroweak corrections," Phys.Rev. D46 (1992) 381-409.

[50] H. E. Haber and D. O'Neil, "Basis-independent methods for the two-Higgsdoublet model III: The CP-conserving limit, custodial symmetry, and the oblique parameters S, T, U," Phys.Rev. D83 (2011) 055017, arXiv:1011.6188 [hep-ph].

[51] M. Baak, M. Goebel, J. Haller, A. Hoecker, and D. et. al.. Kennedy, "The Electroweak Fit of the Standard Model after the Discovery of a New Boson at the LHC," Eur.Phys.J. C72 (2012) 2205, arXiv:1209.2716 [hep-ph].

[52] S. P. Martin and M. T. Vaughn, "Two loop renormalization group equations for soft supersymmetry breaking couplings," Phys.Rev. D50 (1994) 2282 , arXiv:hep-ph/9311340 [hep-ph].

[53] N. Seiberg, "Electric - magnetic duality in supersymmetric nonAbelian gauge theories," Nucl.Phys. B435 (1995) 129-146, arXiv:hep-th/9411149 [hep-th].

[54] K. A. Intriligator and N. Seiberg, "Lectures on supersymmetric gauge theories and electric - magnetic duality," Nucl.Phys.Proc.Suppl. 45BC (1996) 1-28, arXiv:hep-th/9509066 [hep-th].

[55] K. A. Intriligator and B. Wecht, "The Exact superconformal R symmetry maximizes a," Nucl.Phys. B667 (2003) 183-200, arXiv:hep-th/0304128 [hep-th].

[56] M. Strassler, "Generating a fermion mass hierarchy in a composite supersymmetric standard model," Phys.Lett. B376 (1996) 119-126, arXiv:hep-ph/9510342 [hep-ph]. 
[57] S. Chatrchyan et al. [CMS Collaboration], "Search for a standard-model-like higgs boson with a mass in the range 145 to $1000 \mathrm{GeV}$ at the LHC," Eur.Phys.J. C73 (2013) 2469, arXiv:1304.0213 [hep-ex].

[58] N. Craig, J. Galloway, and S. Thomas, "Searching for signs of the second higgs doublet," arXiv:1305.2424 [hep-ph].

[59] J. Fan, M. Reece, and J. T. Ruderman, "Stealth Supersymmetry," JHEP 1111 (2011) 012, arXiv:1105.5135 [hep-ph].

[60] F. Staub, "From Superpotential to Model Files for FeynArts and CalcHep/CompHep," Comput.Phys.Commun. 181 (2010) 1077-1086, arXiv:0909.2863 [hep-ph].

[61] F. Staub, "Automatic Calculation of supersymmetric Renormalization Group Equations and Self Energies," Comput.Phys.Commun. 182 (2011) 808-833, arXiv:1002.0840 [hep-ph]. 